\title{
Analysis of an Adaptive Short-Time Fourier Transform-Based Multicomponent Signal Separation Method Derived from Linear Chirp Local Approximation*
}

\author{
Charles K. Chui ${ }^{1}$, Qingtang Jiang ${ }^{2}$, Lin $\mathrm{Li}^{3}$, and Jian $\mathrm{Lu}^{4}$
}

1. College of Mathematics \& Statistics, Shenzhen University, Shenzhen 518060, China and Department of Mathematics, Hong Kong Baptist University, Hong Kong.

2. Department of Math \& Computer Sci., Univ. of Missouri-St. Louis, St. Louis, MO 63121, USA.

3. School of Electronic Engineering, Xidian University, Xi'an 710071, China.

4. Shenzhen Key Laboratory of Advanced Machine Learning and Applications, College of Mathematics \& Statistics, Shenzhen University, Shenzhen 518060, China.

\begin{abstract}
The synchrosqueezing transform (SST) has been developed as a powerful EMD-like tool for instantaneous frequency (IF) estimation and component separation of non-stationary multicomponent signals. Recently, a direct method of the time-frequency approach, called signal separation operation (SSO), was introduced to solving the problem of multicomponent signal separation. While both SST and SSO are mathematically rigorous on IF estimation, SSO avoids the second step of the two-step SST method in component recovery (mode retrieval). In addition, SSO is simple: the IF of a component is estimated by a time-frequency ridge of the SSO plane; and this component is recovered by simply plugging the time-frequency ridge to the SSO operation. In recent paper "Direct signal separation via extraction of local frequencies with adaptive time-varying parameters", after showing that the SSO operation is related to the adaptive short-time Fourier transform (STFT), the authors obtained a more accurate component recovery formula derived from the linear chirp (also called linear frequency modulation signal) approximation at any local time and they also proposed a recovery scheme to extract
\end{abstract}

${ }^{*}$ This work is partially supported by the Hong Kong Research Council, under Projects $\sharp 12300917$ and $\sharp 12303218$, and HKBU Grants $\sharp$ RC-ICRS/16-17/03 and $\sharp$ RC-FNRA-IG/18-19/SCI/01, the Simons Foundation, under Grant $\sharp 353185$, and the National Natural Science Foundation of China under Grants $\sharp 61972265$ and $\sharp 11871348$, the Natural Science Foundation of Guangdong Province of China under Grant $\sharp 2020$ B1515310008. 
the signal components one by one with the time-varying window updated for each component. However the theoretical analysis of the recovery formula derived from linear chirp local approximation has not been studied there. In this paper, we carry out such analysis and obtain error bounds for IF estimation and component recovery. These results provide a mathematical guarantee to the proposed adaptive STFT-based non-stationary multicomponent signal separation method.

Keywords: Adaptive short-time Fourier transform; signal separation operation; linear chirp local approximation; instantaneous frequency estimation; component recovery; multicomponent signal separation.

AMS Mathematics Subject Classification: 42A38, 42C40, 42C15

\section{Introduction}

Many real-world signals are represented as a superposition of Fourier-like oscillatory modes:

$$
x(t)=A_{0}(t)+\sum_{k=1}^{K} x_{k}(t), \quad x_{k}(t)=A_{k}(t) \cos \left(2 \pi \phi_{k}(t)\right),
$$

where $A_{k}(t), \phi_{k}^{\prime}(t)>0$ and $A_{k}(t)$ varies slowly. (1) is also called an adaptive harmonic model (AHM) representation of $x(t)$, with $A_{0}(t)$ called the trend, $A_{k}(t)$ instantaneous amplitudes (IAs) and $\phi_{k}^{\prime}(t)$ the instantaneous frequencies (IFs). To represent $x(t)$ as (1) is important for extracting information hidden in the non-stationary source signal $x(t)$. The empirical mode decomposition (EMD) scheme is a popular method to decompose non-stationary signals [14]. EMD decomposes a signal into its "IMFs" and "trend" without the concern of recovering the true IMFs and trend of the source signal. In this paper we consider recovering trend $A_{0}(t)$ and components $x_{k}(t)$ of the source signal $x(t)$.

The continuous wavelet transform (CWT)-based synchrosqueezed wavelet transform (SST), introduced in [12] and further developed in [11, provides mathematical theorems to guarantee the recovery of oscillatory modes. Since the seminal work [11] was available to the research community, various SSTs have been proposed and studied, see e.g. [1]-[5], [10], [15]-[18], [21]-[29].

Recently, a direct method of the time-frequency approach, called signal separation operation or signal separation operator (SSO), was introduced in [9] to solving the problem of multicomponent signal separation. While both SST and SSO are mathematically rigorous on IF estimation, SSO avoids the second step of the two-step SST method in component recovery, which depends heavily on the accuracy of the estimated IFs. In addition, SSO is simple: the IF $\phi_{k}^{\prime}(t)$ of the $k$-th 
component $x_{k}(t)$ of a multicomponent signal $x(t)$ is estimated by a time-frequency ridge $\widehat{\theta}_{k}(t)$ of the SSO operation $T_{x}(t, \theta)$; and $x_{k}(t)$ is recovered by simply plugging $\widehat{\theta}_{k}(t)$ to $T_{x}(t, \theta): x_{k}(t) \approx$ $T_{x}\left(t, \widehat{\theta}_{k}(t)\right)$.

In their recent paper [19], the authors show that the SSO operation is related to the adaptive STFT defined by

$$
\begin{aligned}
\widetilde{V}_{x}(t, \eta) & :=\int_{-\infty}^{\infty} x(\tau) g_{\sigma(t)}(\tau-t) e^{-i 2 \pi \eta(\tau-t)} d \tau \\
& =\int_{-\infty}^{\infty} x(t+\tau) \frac{1}{\sigma(t)} g\left(\frac{\tau}{\sigma(t)}\right) e^{-i 2 \pi \eta \tau} d \tau,
\end{aligned}
$$

where $t$ and $\eta$ are the time variable and the frequency variable respectively, $\sigma=\sigma(t)$ is a positive function of $t$, and $g_{\sigma(t)}(\tau)$ is defined by

$$
g_{\sigma(t)}(\tau):=\frac{1}{\sigma(t)} g\left(\frac{\tau}{\sigma(t)}\right)
$$

with $g \in L_{2}(\mathbb{R})$. The window width of $g_{\sigma(t)}(\tau)$ is $\sigma(t)$ (up to a constant), depending on the time variable $t$.

The authors of [19] obtained a more accurate component recovery formula derived from linear chirp (also called linear frequency modulation signal) approximation at any local time and they also proposed a recovery scheme to extract the signal components one by one with the timevarying window updated for each component. However the theoretical analysis of the recovery formula derived from the linear chirp local approximation has not been studied there. In this paper, we carry out such analysis and obtain error bounds for IF estimation and component recovery with this new formula. These results provide a mathematical guarantee to the proposed adaptive STFT-based non-stationary multicomponent signal separation method.

Recently our linear chirp local approximation-based approach has been extended in [20] to 3-dimensional case with variables of time, frequency and chirp rate to recover components with crossover IFs. Very recently the STFT-based SSO method is extended to the continuous wavelet transform ( CWT)-based SSO in [7, 9]. Furthermore, a more accurate component recovery formula based on the adaptive CWT was derived based on linear chirp local approximation in [6].

In this paper we consider the complex version of multi-component signals $x(t)$ of (1):

$$
x(t)=A_{0}(t)+\sum_{k=1}^{K} x_{k}(t)=A_{0}(t)+\sum_{k=1}^{K} A_{k}(t) e^{i 2 \pi \phi_{k}(t)}
$$

with $A_{k}(t), \phi_{k}^{\prime}(t)>0$. For convenience, we let $\phi_{0}(0)=0$. Thus the trend can also be written as $x_{0}(t):=A_{0}(t) e^{i 2 \pi \phi_{0}(t)}$. The main goal of this paper is to analyze the error bound for the recovery formula based on linear chirp approximation (called the linear chirp-based model in this paper). 
To make this paper be self-contained, we also include the error bound for the recovery formula based on sinusoidal signal approximation (called the sinusoidal signal-based model in this paper). The sinusoidal signal-based model and the linear chirp-based model will be studied in Sections 2 and 3 respectively.

Note that when $\sigma(t) \equiv \sigma$, a positive constant, then $\widetilde{V}_{x}(t, \eta)$ in (4) is the conventional (modified) STFT of $x(t)$ with window function $h(t)=\frac{1}{\sigma} g\left(\frac{t}{\sigma}\right)$ :

$$
V_{x}(t, \eta):=\int_{-\infty}^{\infty} x(\tau) h(\tau-t) e^{-i 2 \pi \eta(\tau-t)} d \tau .
$$

Thus our linear chirp local approximation-based recovery formula and its analysis established in Section 3 apply to the conventional STFT-based approach.

\section{Sinusoidal signal-based model}

Let $x(t)$ be a non-stationary multicomponent signal of the form (5). We assume $A_{k}(t), \phi_{k}(t)$ satisfy

$$
\begin{aligned}
& A_{k}(t) \in C^{1}(\mathbb{R}) \cap L_{\infty}(\mathbb{R}), \phi_{k}(t) \in C^{2}(\mathbb{R}), \\
& A_{k}(t)>0, \inf _{t \in \mathbb{R}} \phi_{k}^{\prime}(t)>0, \sup _{t \in \mathbb{R}} \phi_{k}^{\prime}(t)<\infty, \\
& d^{\prime}:=\min _{k \in\{2, \cdots, K\}} \min _{t \in \mathbb{R}}\left(\phi_{k}^{\prime}(t)-\phi_{k-1}^{\prime}(t)\right)>0 .
\end{aligned}
$$

In this section we consider the case that each component $x_{k}(t)=A_{k}(t) e^{i 2 \pi \phi_{k}(t)}$ is approximated locally by a sinusoidal signal. More precisely, we assume $A_{k}(t)$ and $\phi_{k}^{\prime}(t)$ changes slowly:

$$
\begin{aligned}
& \left|A_{k}(t+\tau)-A_{k}(t)\right| \leq \varepsilon_{1}|\tau| A_{k}(t), t \in \mathbb{R}, 0 \leq k \leq K, \\
& \left|\phi_{k}^{\prime \prime}(t)\right| \leq \varepsilon_{2}, t \in \mathbb{R}, 1 \leq k \leq K,
\end{aligned}
$$

for some positive number $\varepsilon_{1}, \varepsilon_{2}$. Let $\mathcal{B}_{\varepsilon_{1}, \varepsilon_{2}}$ denote the set of multicomponent signals of (5) satisfying (7)-(11).

Denote

$$
\mu=\mu(t):=\min _{0 \leq k \leq K} A_{k}(t), \quad M=M(t):=\sum_{k=0}^{K} A_{k}(t), \quad M_{\ell}=M_{\ell}(t):=\sum_{k \neq \ell} A_{k}(t) .
$$

Throughout this paper, $\sum_{k \neq \ell}$ denotes $\sum_{\{k: k \neq \ell, 0 \leq k \leq K\}}$.

Let $x(t) \in \mathcal{B}_{\varepsilon_{1}, \varepsilon_{2}}$. Write $x_{k}(t+\tau)$ as

$$
x_{k}(t+\tau)=x_{k}(t) e^{i 2 \pi \phi_{k}^{\prime}(t) \tau}+x_{k, \mathrm{rs}}(t, \tau)
$$


where $x_{k, \text { rs }}(t, \tau):=x_{k}(t+\tau)-x_{k}(t) e^{i 2 \pi \phi_{k}^{\prime}(t) \tau}$. Note that for any given $t, x_{k}(t) e^{i 2 \pi \phi_{k}^{\prime}(t) \tau}$ (as a function of $\tau$ ) is a sinusoidal function in $\tau$. We use $x_{k}(t) e^{i 2 \pi \phi_{k}^{\prime}(t) \tau}$ to approximate $x(t+\tau)$ at a local time $t$. By $(13)$, we have

$$
\begin{aligned}
\widetilde{V}_{x}(t, \eta) & =\int_{\mathbb{R}} \sum_{k=0}^{K}\left(x_{k}(t) e^{i 2 \pi \phi_{k}^{\prime}(t) \tau}+x_{k, \mathrm{rs}}(t, \tau)\right) \frac{1}{\sigma(t)} g\left(\frac{\tau}{\sigma(t)}\right) e^{-i 2 \pi \eta \tau} d \tau \\
& =\sum_{k=0}^{K} x_{k}(t) \widehat{g}\left(\sigma(t)\left(\eta-\phi_{k}^{\prime}(t)\right)+\operatorname{rem}_{0},\right.
\end{aligned}
$$

where $\operatorname{rem}_{0}$ is the remainder for the expansion of $\widetilde{V}_{x}(t, \eta)$ in 14 given by

$$
\operatorname{rem}_{0}:=\sum_{k=0}^{K} \int_{\mathbb{R}} x_{k, \mathrm{rs}}(t, \tau) \frac{1}{\sigma(t)} g\left(\frac{\tau}{\sigma(t)}\right) e^{-i 2 \pi \eta \tau} d \tau
$$

Writing $x_{k, \mathrm{rs}}(t, \tau)$ as

$$
x_{k, \mathrm{rs}}(t, \tau)=\left(A_{k}(t+\tau)-A_{k}(t)\right) e^{i 2 \pi \phi_{k}(t+\tau)}+x_{k}(t) e^{i 2 \pi \phi_{k}^{\prime}(t) \tau}\left(e^{i 2 \pi\left(\phi_{k}(t+\tau)-\phi_{k}(t)-\phi_{k}^{\prime}(t) \tau\right)}-1\right) .
$$

From (11), we have

$$
\left|e^{i 2 \pi\left(\phi_{k}(t+\tau)-\phi_{k}(t)-\phi_{k}^{\prime}(t) \tau\right)}-1\right| \leq 2 \pi\left|\phi_{k}(t+\tau)-\phi_{k}(t)-\phi_{k}^{\prime}(t) \tau\right| \leq \pi \varepsilon_{2}|\tau|^{2} .
$$

This, together with 10 , leads to

$$
\begin{aligned}
\left|\mathrm{rem}_{0}\right| & \leq \sum_{k=0}^{K} \int_{\mathbb{R}} \varepsilon_{1}|\tau| A_{k}(t) \frac{1}{\sigma(t)}\left|g\left(\frac{\tau}{\sigma(t)}\right)\right| d \tau+\sum_{k=1}^{K} A_{k}(t) \int_{\mathbb{R}} \pi \varepsilon_{2}|\tau|^{2} \frac{1}{\sigma(t)}\left|g\left(\frac{\tau}{\sigma(t)}\right)\right| d \tau \\
& =M(t)\left(\varepsilon_{1} I_{1} \sigma(t)+\pi \varepsilon_{2} I_{2} \sigma^{2}(t)\right)=: M(t) \lambda_{0}(t),
\end{aligned}
$$

where $I_{n}$ and $\lambda_{0}(t)$ are defined by

$$
\begin{aligned}
& I_{n}:=\int_{\mathbb{R}}\left|\tau^{n} g(\tau)\right| d \tau, \\
& \lambda_{0}(t):=\varepsilon_{1} I_{1} \sigma(t)+\pi \varepsilon_{2} I_{2} \sigma^{2}(t) .
\end{aligned}
$$

If the remainder $\operatorname{rem}_{0}$ in (14) is small, then the term $x_{k}(t) \widehat{g}\left(\sigma(t)\left(\eta-\phi_{k}^{\prime}(t)\right)\right.$ in 14 governs the time-frequency zone of the STFT $\widetilde{V}_{x_{k}}$ of the $k$ th component $x_{k}(t)$ of $x(t)$. In particular, if $g$ is band-limited, that is $\widehat{g}$ is compactly supported, to say $\operatorname{supp}(\widehat{g}) \subset[-\triangle, \triangle]$ for some $\triangle>0$, then $x_{k}(t) \widehat{g}\left(\sigma(t)\left(\eta-\phi_{k}^{\prime}(t)\right)\right.$ lies within the time-frequency zone:

$$
\left\{(t, \eta):\left|\eta-\phi_{k}^{\prime}(t)\right|<\frac{\triangle}{\sigma(t)}, t \in \mathbb{R}\right\}
$$


If $\widehat{g}$ is not compactly supported, we need to define the essential support of $\widehat{g}$ outside which $\widehat{g}(\xi) \approx 0$. More precisely, for a given threshold $0<\tau_{0}<1$, if $|\widehat{g}(\xi)| \leq \tau_{0}$ for $|\xi| \geq \alpha$, then we say $\widehat{g}(\xi)$ is essentially supported in $[-\alpha, \alpha]$. When $|\widehat{g}(\xi)|$ is even and decreasing for $\xi \geq 0$, then $\alpha$ can be obtained by solving

$$
|\widehat{g}(\alpha)|=\tau_{0}
$$

For example, when $g$ is the Gaussian window function given by

$$
g(t)=\frac{1}{\sqrt{2 \pi}} e^{-\frac{t^{2}}{2}}
$$

then, with $\widehat{g}(\xi)=e^{-2 \pi^{2} \xi^{2}}$, the corresponding $\alpha$ is given by

$$
\alpha=\frac{1}{2 \pi} \sqrt{2 \ln \left(\frac{1}{\tau_{0}}\right)}
$$

In the following we assume $|\widehat{g}(\xi)|$ is even. In addition, we assume $|\widehat{g}(\xi)|$ decreasing for $\xi \geq 0$ if $\widehat{g}(\xi)$ is not compactly supported, and we let $|\widehat{g}|^{-1}$ denote the inverse function of $|\widehat{g}(\xi)|, \xi \in(0, \infty)$. If supp $\widehat{g}=[-\beta, \beta]$ for some $\beta>0$, then we assume $|\widehat{g}(\xi)|$ is decreasing for $\xi \in[0, \beta]$, and let $|\widehat{g}|^{-1}$ denote the inverse function of $|\widehat{g}(\xi)|, \xi \in(0, \beta)$. Moreover, we assume

$$
\int_{\mathbb{R}} g(t) d t=1
$$

For $g$ with $\widehat{g}(\xi)$ essentially supported in $[-\alpha, \alpha]$, we then define the time-frequency zone $Z_{k}$ of the $k$ th-component $x_{k}(t)$ of $x(t)$ by

$$
Z_{k}:=\left\{(t, \eta): \mid \widehat{g}\left(\sigma(t)\left(\eta-\phi_{k}^{\prime}(t)\right) \mid>\tau_{0}, t \in \mathbb{R}\right\}=\left\{(t, \eta):\left|\eta-\phi_{k}^{\prime}(t)\right|<\frac{\alpha}{\sigma(t)}, t \in \mathbb{R}\right\}\right.
$$

Thus the multicomponent signal $x(t)$ is well-separated, if $\sigma(t)$ satisfies

$$
\sigma(t) \geq \frac{2 \alpha}{\phi_{k}^{\prime}(t)-\phi_{k-1}^{\prime}(t)}, t \in \mathbb{R}, k=1,2, \cdots, K .
$$

In this case $Z_{k} \cap Z_{\ell}=\emptyset, k \neq \ell$. In this section we assume that 22 holds for some $\sigma(t)$. Due to (9), there always exists a bounded $\sigma(t)$ such that $(22)$ holds. When $g$ is the Gaussian window function, for the sinusoidal signal-based adaptive method, we may choose $\sigma(t)$ as in [17] to be

$$
\sigma_{1}(t):=\max _{2 \leq k \leq K}\left\{\frac{2 \alpha}{\phi_{k}^{\prime}(t)-\phi_{k-1}^{\prime}(t)}\right\}=\frac{2 \alpha}{\min _{2 \leq k \leq K}\left\{\phi_{k}^{\prime}(t)-\phi_{k-1}^{\prime}(t)\right\}} .
$$

The reason is that from the error bound for the recovery formula (see Corollary 2 below), the smaller $\sigma(t)$ is, the smaller the error bound.

From 22, we have

$$
\sigma(t)\left|\phi_{k}^{\prime}(t)-\phi_{\ell}^{\prime}(t)\right| \geq 2 \alpha|k-\ell| .
$$


and hence for $(t, \eta) \in Z_{k}$,

$$
\left|\eta-\phi_{\ell}^{\prime}(t)\right| \geq\left|\phi_{\ell}^{\prime}(t)-\phi_{k}^{\prime}(t)\right|-\left|\eta-\phi_{k}^{\prime}(t)\right| \geq \frac{\alpha(2|\ell-k|-1)}{\sigma(t)} .
$$

Denote

$$
\operatorname{err}_{\ell}=\operatorname{err}_{\ell}(t):=M(t) \lambda_{0}(t)+\sum_{k \neq \ell} A_{k}(t) \mid \widehat{g}(\alpha(2|\ell-k|-1) \mid .
$$

Clearly, we have

$$
\operatorname{err}_{\ell} \leq M(t) \lambda_{0}(t)+\tau_{0} \sum_{k \neq \ell} A_{k}(t)
$$

For a fixed $t$, and a positive number $\widetilde{\epsilon}_{1}$, we let $\mathcal{G}_{t}$ and $\mathcal{G}_{t, k}$ denote the sets defined by

$$
\mathcal{G}_{t}:=\left\{\eta:\left|\widetilde{V}_{x}(t, \eta)\right|>\widetilde{\epsilon}_{1}\right\}, \mathcal{G}_{t, k}:=\left\{\eta \in \mathcal{G}_{t}:\left|\eta-\phi_{k}^{\prime}(t)\right|<\frac{\alpha}{\sigma(t)}\right\} .
$$

Note that $\mathcal{G}_{t}$ and $\mathcal{G}_{t, k}$ depend on $\widetilde{\epsilon}_{1}$, and for simplicity of presentation, we drop $\widetilde{\epsilon}_{1}$ from them. Also observe that $\mathcal{G}_{t, k}=\mathcal{G}_{t} \cap\left\{\eta:(t, \eta) \in Z_{k}\right\}$. Denote

$$
\widehat{\eta}_{0}:=0, \widehat{\eta}_{\ell}=\widehat{\eta}_{\ell}(t):=\operatorname{argmax}_{\eta \in \mathcal{G}_{t, \ell}}\left|\widetilde{V}_{x}(t, \eta)\right|, \quad \ell=1, \cdots, K \text {. }
$$

Theorem 1. Let $x(t) \in \mathcal{B}_{\varepsilon_{1}, \varepsilon_{2}}$ for some $\varepsilon_{1}, \varepsilon_{2}>0$ and $g$ be a window function. Suppose $\sigma(t)>0$ satisfies 22, and that

$$
2 M(t)\left(\tau_{0}+\lambda_{0}(t)\right) \leq \mu(t) .
$$

Let $\widetilde{\epsilon}_{1}=\widetilde{\epsilon}_{1}(t)>0$ be a function satisfying

$$
M(t)\left(\tau_{0}+\lambda_{0}(t)\right) \leq \widetilde{\epsilon}_{1} \leq \mu(t)-M(t)\left(\tau_{0}+\lambda_{0}(t)\right) .
$$

Then the following statements hold.

(a) Let $\mathcal{G}_{t}$ and $\mathcal{G}_{t, k}$ be the sets defined by (27) for some $\widetilde{\epsilon}_{1}$ satisfying (30). Then $\mathcal{G}_{t}$ can be expressed as a disjoint union of exactly $K+1$ non-empty sets $\mathcal{G}_{t, k}, 0 \leq k \leq K$.

(b) Let $\widehat{\eta}_{\ell}$ be defined by (28). Then for $\ell=1, \cdots, K$,

$$
\left|\widehat{\eta}_{\ell}(t)-\phi_{\ell}^{\prime}(t)\right| \leq \mathrm{bd}_{1, \ell}:=\frac{1}{\sigma(t)}|\widehat{g}|^{-1}\left(1-\frac{2 \operatorname{err}_{\ell}(t)}{A_{\ell}(t)}\right)
$$

where $\operatorname{err}_{\ell}$ is defined by (25).

(c) For $\ell=0,1, \cdots, K$,

$$
\left|\widetilde{V}_{x}\left(t, \widehat{\eta}_{\ell}\right)-x_{\ell}(t)\right| \leq \mathrm{bd}_{2, \ell}:=\operatorname{err}_{\ell}(t)+2 \pi I_{1} A_{\ell}(t)|\widehat{g}|^{-1}\left(1-\frac{2 \operatorname{err}_{\ell}(t)}{A_{\ell}(t)}\right) .
$$


(d) If in addition the window function $g(t) \geq 0$ for $t \in \mathbb{R}$, then for $\ell=0,1, \cdots, K$,

$$
|| \widetilde{V}_{x}\left(t, \widehat{\eta}_{\ell}\right)\left|-A_{\ell}(t)\right| \leq \operatorname{err}_{\ell}(t)
$$

Note (29) holds if $\varepsilon_{1}, \varepsilon_{2}, \tau_{0}$ are small enough. (33) gives an error bound for IA and trend estimate

$$
A_{\ell}(t) \approx\left|\widetilde{V}_{x}\left(t, \widehat{\eta}_{\ell}\right)\right|
$$

The proof of Theorem 1 will delayed to the end of this section.

Remark 1. Here we remark that $\operatorname{err}_{\ell}(t)<\frac{1}{2} A_{\ell}(t)$ if $2 M(t)\left(\tau_{0}+\lambda_{0}(t)\right) \leq \mu(t)$, and hence, $|\widehat{g}|^{-1}\left(1-\frac{2 \mathrm{err}_{\ell}(t)}{A_{\ell}(t)}\right)$ is well defined. Indeed, from 26 ,

$$
\begin{aligned}
\operatorname{err}_{\ell}(t) & \leq M(t) \lambda_{0}(t)+\tau_{0} \sum_{k \neq \ell} A_{k}(t) \leq \frac{1}{2} \mu(t)-M(t) \tau_{0}+\tau_{0} \sum_{k \neq \ell} A_{k}(t) \\
& <\frac{1}{2} A_{\ell}(t)-M(t) \tau_{0}+\tau_{0} \sum_{k=1}^{K} A_{k}(t)=\frac{1}{2} A_{\ell}(t) .
\end{aligned}
$$

When $\widehat{g}(\xi)$ is supported in $[-\alpha, \alpha]$, we can set $\tau_{0}$ in Theorem 1 to be zero. Thus the condition in 29 is reduced to $2 M(t) \lambda_{0}(t) \leq \mu(t)$. In addition, the error $\operatorname{err}_{\ell}(t)$ in 25 is simply $M(t) \lambda_{0}(t)$. To summarize, we have the following corollary.

Corollary 1. Let $x(t) \in \mathcal{B}_{\varepsilon_{1}, \varepsilon_{2}}$ for some $\varepsilon_{1}, \varepsilon_{2}>0$ and $g$ be a window function with supp $(\widehat{g}) \subseteq$ $[-\alpha, \alpha]$. Suppose $\sigma(t)>0$ satisfies $(22)$. If $\varepsilon_{1}, \varepsilon_{2}$ are small enough such that $2 M(t) \lambda_{0}(t) \leq \mu(t)$, then we have the following.

(a) Let $\mathcal{G}_{t}$ and $\mathcal{G}_{t, k}$ be the sets defined by (27) for some $\widetilde{\epsilon}_{1}>0$. If $\widetilde{\epsilon}_{1}$ satisfies $M(t) \lambda_{0}(t) \leq \widetilde{\epsilon}_{1} \leq$ $\mu(t)-M(t) \lambda_{0}(t)$, then $\mathcal{G}_{t}$ can be expressed as a disjoint union of exactly $K+1$ non-empty sets $\mathcal{G}_{t, k}, 0 \leq k \leq K$.

(b) Let $\widehat{\eta}_{\ell}$ be defined by (28). Then for $\ell=1,2, \cdots, K$,

$$
\left|\widehat{\eta}_{\ell}(t)-\phi_{\ell}^{\prime}(t)\right| \leq \frac{1}{\sigma(t)}|\widehat{g}|^{-1}\left(1-\frac{2 M(t) \lambda_{0}(t)}{A_{\ell}(t)}\right) .
$$

(c) For $\ell=0,1, \cdots, K$,

$$
\left|\widetilde{V}_{x}\left(t, \widehat{\eta}_{\ell}\right)-x_{\ell}(t)\right| \leq M(t) \lambda_{0}(t)+2 \pi I_{1} A_{\ell}(t)|\widehat{g}|^{-1}\left(1-\frac{2 M(t) \lambda_{0}(t)}{A_{\ell}(t)}\right) .
$$

(d) If in addition the window function $g(t) \geq 0$ for $t \in \mathbb{R}$, then for $\ell=0,1, \cdots, K$,

$$
|| \widetilde{V}_{x}\left(t, \widehat{\eta}_{\ell}\right)\left|-A_{\ell}(t)\right| \leq M(t) \lambda_{0}(t) .
$$


Next let us consider the case that $g(t)$ is the Gaussian window function given by (19). In this case $\widehat{g}(\xi)=e^{-2 \pi^{2} \xi^{2}}$. Thus $\operatorname{err}_{\ell}(t)$ defined by 25 is

$$
\operatorname{err}_{\ell}(t)=\varepsilon_{1} I_{1} \sigma(t) M(t)+\pi \varepsilon_{2} I_{2} \sigma^{2}(t) M(t)+\sum_{k \neq \ell} A_{k}(t) e^{-2 \pi^{2}(\alpha(2|\ell-k|-1))^{2}} .
$$

For this $g$, we have

$$
|\widehat{g}|^{-1}(\xi)=\widehat{g}(\xi)^{-1}=\frac{1}{\pi \sqrt{2}} \sqrt{-\ln \xi}, 0<\xi<1 .
$$

Hence the error bound $b_{1, \ell}$ in $(31)$ is

$$
\mathrm{bd}_{1, \ell}=\frac{1}{\sigma(t)}|\widehat{g}|^{-1}\left(1-\frac{2 \operatorname{err}_{\ell}(t)}{A_{\ell}(t)}\right)=\frac{1}{\sigma(t) \pi \sqrt{2}} \sqrt{-\ln \left(1-\frac{2 \operatorname{err}_{\ell}(t)}{A_{\ell}(t)}\right)} .
$$

Using the fact $-\ln (1-t)<2 t$ for $0<t<\frac{1}{2}$ and assuming $\varepsilon_{1}, \varepsilon_{2}, \tau_{0}$ are small enough such that $\frac{2 \mathrm{err}_{\ell}(t)}{A_{\ell}(t)}<\frac{1}{2}$, we have

$$
\operatorname{bd}_{1, \ell}<\frac{\sqrt{2}}{\sigma(t) \pi} \sqrt{\frac{\operatorname{err}_{\ell}(t)}{A_{\ell}(t)}}
$$

In this case the error bound $\mathrm{bd}_{2, \ell}$ in 32 for component recovery satisfies

$$
\operatorname{bd}_{2, \ell}<\operatorname{err}_{\ell}(t)+2 \sqrt{2} I_{1} \sqrt{A_{\ell}(t) \operatorname{err}_{\ell}(t)} .
$$

To summarize, we have the following corollary.

Corollary 2. Let $x(t) \in \mathcal{B}_{\varepsilon_{1}, \varepsilon_{2}}$ for some $\varepsilon_{1}, \varepsilon_{2}>0$. Suppose the conditions in Theorem 1 for the Gaussian window function $g$ given by (19) are satisfied and that $\operatorname{err}_{\ell}(t)<\frac{1}{4} A_{\ell}(t)$. Then Part (a) of Theorem 1 holds; and with $\widehat{\eta}_{\ell}$ defined by (28), we have

$$
\begin{aligned}
& \left|\widehat{\eta}_{\ell}(t)-\phi_{\ell}^{\prime}(t)\right|<\frac{\sqrt{2}}{\sigma(t) \pi} \sqrt{\frac{\operatorname{err}_{\ell}(t)}{A_{\ell}(t)}}, \quad \ell=1,2, \cdots, K, \\
& \left|\widetilde{V}_{x}\left(t, \widehat{\eta}_{\ell}\right)-x_{\ell}(t)\right|<\operatorname{err}_{\ell}(t)+2 \sqrt{2} I_{1} \sqrt{A_{\ell}(t) \operatorname{err}_{\ell}(t)}, \quad \ell=0,1, \cdots, K, \\
& || \widetilde{V}_{x}\left(t, \widehat{\eta}_{\ell}\right)\left|-A_{\ell}(t)\right| \leq \operatorname{err}_{\ell}(t), \quad \ell=0,1, \cdots, K,
\end{aligned}
$$

where $\operatorname{err}_{\ell}(t)$ is defined by (37).

Remark 2. Observe that a smaller $\sigma(t)$ results in smaller error bounds in (39) and 40) for component recovery and instantaneous amplitude recovery respectively. Note that the IF estimate error bound in $(38)$ is

$$
\frac{\sqrt{2}}{\sigma(t) \pi} \sqrt{\frac{\operatorname{err}_{\ell}(t)}{A_{\ell}(t)}}=\frac{\sqrt{2}}{\pi}\left(\frac{M(t)}{A_{\ell}(t) \sigma(t)} \varepsilon_{1} I_{1}+\frac{M(t)}{A_{\ell}(t)} \pi \varepsilon_{2} I_{2}+\frac{1}{\sigma^{2}(t) A_{\ell}(t)} \sum_{k \neq \ell} A_{k}(t) e^{-2 \pi^{2}(\alpha(2|\ell-k|-1))^{2}}\right)^{1 / 2} .
$$

Thus for IF estimate, a larger $\sigma(t)$ actually gives a smaller error bound. Of course $\sigma(t)$ cannot be arbitrarily large due to the restriction in 29. 
Finally, in this section, we give the proof of Theorem 1 Here we consider the case $g$ is nonbandlimited. The proof of Theorem 1 when $g$ is bandlimited, which is Corollary 1 , is the same but simpler.

Proof of Theorem 1(a). Clearly $\cup_{k=0}^{K} \mathcal{G}_{t, k} \subseteq \mathcal{G}_{t}$. Next we show $\mathcal{G}_{t} \subseteq \cup_{k=0}^{K} \mathcal{G}_{t, k}$. Let $\eta \in \mathcal{G}_{t}$. Assume $\eta \notin \cup_{k=0}^{K} \mathcal{G}_{t, k}$. That is $(t, \eta) \notin \cup_{k=0}^{K} Z_{k}$. Then by the definition of $Z_{k}$ in (21), we have $\left|\widehat{g}\left(\sigma(t)\left(\eta-\phi_{k}^{\prime}(t)\right)\right)\right| \leq \tau_{0}$. Hence, by (14) and 15 , we have

$$
\begin{aligned}
\left|\widetilde{V}_{x}(t, \eta)\right| & \leq \sum_{k=0}^{K}\left|x_{k}(t) \widehat{g}\left(\sigma(t)\left(\eta-\phi_{k}^{\prime}(t)\right)\right)\right|+\left|\mathrm{rem}_{0}\right| \\
& \leq \tau_{0} \sum_{k=0}^{K} A_{k}(t)+M(t) \lambda_{0}(t) \leq \widetilde{\epsilon}_{1},
\end{aligned}
$$

a contradiction to the assumption $\left|\widetilde{V}_{x}(t, \eta)\right|>\widetilde{\epsilon}_{1}$. Thus $(t, \eta) \in Z_{\ell}$ for some $\ell$. This shows that $\eta \in \mathcal{G}_{t, \ell}$. Hence $\mathcal{G}_{t}=\cup_{k=0}^{K} \mathcal{G}_{t, k}$. Since $Z_{k}, 0 \leq k \leq K$ are not overlapping, we know $\mathcal{G}_{t, k}, 0 \leq k \leq K$ are disjoint.

To show that $\mathcal{G}_{t, \ell}$ is non-empty, it is enough to show $\phi_{\ell}^{\prime}(t) \in \mathcal{G}_{t}$ which implies $\phi_{\ell}^{\prime}(t) \in \mathcal{G}_{\ell, t}$ since $\left(t, \phi^{\prime}{ }_{k}(t)\right) \in Z_{k}$. From

$$
\begin{aligned}
\left|\widetilde{V}_{x}\left(t, \phi_{\ell}^{\prime}(t)\right)\right| & \geq \sum_{k=0}^{K}\left|x_{k}(t) \widehat{g}\left(\sigma(t)\left(\phi_{\ell}^{\prime}(t)-\phi_{k}^{\prime}(t)\right)\right)\right|-\left|\operatorname{rem}_{0}\right| \\
& \geq\left|x_{\ell}(t) \widehat{g}(0)\right|-\sum_{k \neq \ell}\left|x_{k}(t)\right| \tau_{0}-M(t) \lambda_{0}(t) \\
& \geq A_{\ell}(t)-M(t) \tau_{0}-M(t) \lambda_{0}(t) \\
& \geq \mu(t)-M(t)\left(\tau_{0}+\lambda_{0}(t)\right) \geq \widetilde{\epsilon}_{1}
\end{aligned}
$$

we conclude that $\phi_{\ell}^{\prime}(t) \in \mathcal{G}_{t}$. Therefore, the statements in (a) hold.

Proof of Theorem 1(b). For any $\eta \in \mathcal{G}_{t, \ell}$, from (14), we have

$$
\begin{aligned}
& \mid \widetilde{V}_{x}(t, \eta)-x_{\ell}(t) \widehat{g}\left(\sigma(t)\left(\eta-\phi_{\ell}^{\prime}(t)\right) \mid\right. \\
& =\mid \sum_{k \neq \ell} x_{k}(t) \widehat{g}\left(\sigma(t)\left(\eta-\phi_{k}^{\prime}(t)\right)+\mathrm{rem}_{0} \mid\right. \\
& \leq \sum_{k \neq \ell} A_{k}(t) \mid \widehat{g}\left(\alpha(2|\ell-k|-1) \mid+M(t) \lambda_{0}(t),\right.
\end{aligned}
$$

where the last inequality follows from (15), 24) and the assumption that $|\widehat{g}(\xi)|$ is decreasing for $\xi \geq 0$. Thus

$$
\mid \widetilde{V}_{x}(t, \eta)-x_{\ell}(t) \widehat{g}\left(\sigma(t)\left(\eta-\phi_{\ell}^{\prime}(t)\right) \mid \leq \operatorname{err}_{\ell}(t)\right.
$$


Hence, letting $\eta=\phi_{\ell}^{\prime}(t)$, we have

$$
\left|\widetilde{V}_{x}\left(t, \phi_{\ell}^{\prime}(t)\right)\right| \geq\left|x_{\ell}(t) \widehat{g}(0)\right|-\operatorname{err}_{\ell}(t)=A_{\ell}(t)-\operatorname{err}_{\ell}(t),
$$

since $\widehat{g}(0)=1$. By the definition of $\widehat{\eta}_{\ell}$ and (41) again, we have

$$
\left|\widetilde{V}_{x}\left(t, \phi_{\ell}^{\prime}(t)\right)\right| \leq\left|\widetilde{V}_{x}\left(t, \widehat{\eta}_{\ell}\right)\right| \leq \mid x_{\ell}(t) \widehat{g}\left(\sigma(t)\left(\widehat{\eta}_{\ell}-\phi_{\ell}^{\prime}(t)\right) \mid+\operatorname{err}_{\ell}(t)\right.
$$

This, together with (42), implies

$$
A_{\ell}(t)-\operatorname{err}_{\ell}(t) \leq A_{\ell}(t) \mid \widehat{g}\left(\sigma(t)\left(\widehat{\eta}_{\ell}-\phi_{\ell}^{\prime}(t)\right) \mid+\operatorname{err}_{\ell}(t),\right.
$$

or equivalently

$$
0<1-\frac{2 \operatorname{err}_{\ell}(t)}{A_{\ell}(t)} \leq \mid \widehat{g}\left(\sigma(t)\left(\widehat{\eta}_{\ell}-\phi_{\ell}^{\prime}(t)\right) \mid\right.
$$

Then (31) follows from the above inequality and that $|\widehat{g}(\xi)|$ is decreasing on $(0, \infty)$.

Proof of Theorem 1(c). From (41), we have

$$
\begin{aligned}
& \left|\widetilde{V}_{x}\left(t, \widehat{\eta}_{\ell}\right)-x_{\ell}(t)\right| \\
& \leq \mid \widetilde{V}_{x}\left(t, \widehat{\eta}_{\ell}\right)-x_{\ell}(t) \widehat{g}\left(\sigma ( t ) ( \widehat { \eta } _ { \ell } - \phi _ { \ell } ^ { \prime } ( t ) ) | + | x _ { \ell } ( t ) \widehat { g } \left(\sigma(t)\left(\widehat{\eta}_{\ell}-\phi_{\ell}^{\prime}(t)\right)-x_{\ell}(t) \mid\right.\right. \\
& \leq \operatorname{err}_{\ell}(t)+A_{\ell}(t)\left|\int_{\mathbb{R}} g(\tau)\left(e^{-i 2 \pi \sigma(t)\left(\widehat{\eta}_{\ell}-\phi_{\ell}^{\prime}(t)\right) \tau}-1\right) d \tau\right| \\
& \leq \operatorname{err}_{\ell}(t)+A_{\ell}(t) \int_{\mathbb{R}}|g(\tau)| 2 \pi \sigma(t)\left|\widehat{\eta}_{\ell}-\phi_{\ell}^{\prime}(t)\right||\tau| d \tau \\
& \leq \operatorname{err}_{\ell}(t)+A_{\ell}(t) 2 \pi|\widehat{g}|^{-1}\left(1-\frac{2 \operatorname{err}_{\ell}(t)}{A_{\ell}(t)}\right) \int_{\mathbb{R}}|g(\tau)||\tau| d \tau \\
& \leq \operatorname{err}_{\ell}(t)+2 \pi I_{1} A_{\ell}(t)|\widehat{g}|^{-1}\left(1-\frac{2 \operatorname{err}_{\ell}(t)}{A_{\ell}(t)}\right) .
\end{aligned}
$$

This shows 32 .

Proof of Theorem 1)(d). By 42, we have

$$
\left|\widetilde{V}_{x}\left(t, \widehat{\eta}_{\ell}\right)\right| \geq\left|\widetilde{V}_{x}\left(t, \phi_{\ell}^{\prime}(t)\right)\right| \geq A_{\ell}(t)-\operatorname{err}_{\ell}(t) .
$$

On the other hand, when $g(t) \geq 0$, we have $|\widehat{g}(\xi)| \leq 1$ for any $\xi \in \mathbb{R}$. This fact and 41] imply

$$
\left|\widetilde{V}_{x}\left(t, \widehat{\eta}_{\ell}\right)\right| \leq \mid x_{\ell}(t) \widehat{g}\left(\sigma(t)\left(\widehat{\eta}_{\ell}-\phi_{\ell}^{\prime}(t)\right) \mid+\operatorname{err}_{\ell}(t) \leq A_{\ell}(t)+\operatorname{err}_{\ell}(t) .\right.
$$

(33) follows from (43) and 44). This completes the proof of Theorem 1(d). 


\section{$3 \quad$ Linear chirp-based model}

We consider multicomponent signals $x(t)$ of (5) with $A_{k}(t)$ satisfying $(10)$ and

$$
\begin{aligned}
& \phi_{k}(t) \in C^{3}(\mathbb{R}), \phi_{k}^{\prime \prime}(t) \in L_{\infty}(\mathbb{R}), \\
& \left|\phi_{k}^{(3)}(t)\right| \leq \varepsilon_{3}, t \in \mathbb{R}, 1 \leq k \leq K
\end{aligned}
$$

for some positive number $\varepsilon_{3}$. As shown below, conditions 10 and 46 imply that each component $x_{k}(t)$ is well approximated locally by linear chirps. We say $s(t)$ is a linear chirp (also called a linear frequency modulated (LFM) signal) if

$$
s(t)=A e^{i 2 \pi \phi(t)}=A e^{i 2 \pi\left(c t+\frac{1}{2} r t^{2}\right)},
$$

for some constants $c, r$ with $r \neq 0$.

For a given $t$, we use $G_{k}(\xi)$ to denote the Fourier transform of $e^{i \pi \sigma(t) \phi_{k}^{\prime \prime}(t) \tau^{2}} g(\tau), \tau \in \mathbb{R}$, namely,

$$
G_{k}(\xi)=G_{k, t}(\xi):=\int_{\mathbb{R}} e^{i \pi \sigma^{2}(t) \phi_{k}^{\prime \prime}(t) \tau^{2}} g(\tau) e^{-i 2 \pi \xi \tau} d \tau
$$

Note that $G_{k}(\xi)$ depends on $t$ also if $\phi_{k}^{\prime \prime}(t) \neq 0$. We drop $t$ in $G_{k, t}$ for simplicity. For a window function $g$, define

$$
\breve{g}(\xi, \lambda):=\mathcal{F}\left(e^{-i \pi \lambda \tau^{2}} g(\tau)\right)(\xi)=\int_{\mathbb{R}} g(\tau) e^{-i 2 \pi \xi \tau-i \pi \lambda \tau^{2}} d \tau .
$$

Definition 1. (Admissible window function) A window function $g \in L_{1}(\mathbb{R}) \cap L_{2}(\mathbb{R})$ is called an admissible window function if for any $\lambda \in \mathbb{R},|\breve{g}(\xi, \lambda)|$ is even in $\xi$ and is decreasing for $\xi \geq 0$.

If $g$ is an admissible window function, then for $G_{k}(\xi)$ defined by $(48),\left|G_{k}(\xi)\right|$ is even and decreasing for $\xi \geq 0$. We use $\left|G_{k}\right|^{-1}(\xi)$ to denote the inverse function of $\left|G_{k}(\xi)\right|, \xi \geq 0$. In this section, we assume window function $g$ is admissible.

For each component $x_{k}(t)=A_{k}(t) e^{i 2 \pi \phi_{k}(t)}$ in (5), $0 \leq k \leq K$, we write $x_{k}(t+\tau)$ as

$$
x_{k}(t+\tau)=x_{k}(t) e^{i 2 \pi\left(\phi_{k}^{\prime}(t) \tau+\frac{1}{2} \phi_{k}^{\prime \prime}(t) \tau^{2}\right)}+x_{k, \mathrm{rl}}(t, \tau),
$$

where $x_{k, \mathrm{rl}}(t, \tau)=x_{k}(t+\tau)-x_{k}(t) e^{i 2 \pi\left(\phi_{k}^{\prime}(t) \tau+\frac{1}{2} \phi_{k}^{\prime \prime}(t) \tau^{2}\right)}$. Note that for a fixed $t, x_{k}(t) e^{i 2 \pi\left(\phi_{k}^{\prime}(t) \tau+\frac{1}{2} \phi_{k}^{\prime \prime}(t) \tau^{2}\right)}$ is a linear chirp in $\tau$ (assuming $\phi_{k}^{\prime \prime}(t) \neq 0$ ). We use $x_{k}(t) e^{i 2 \pi\left(\phi_{k}^{\prime}(t) \tau+\frac{1}{2} \phi_{k}^{\prime \prime}(t) \tau^{2}\right)}$ to approximate $x(t+\tau)$ 
at a local time $t$ in the following way:

$$
\begin{aligned}
\widetilde{V}_{x}(t, \eta) & =\sum_{k=0}^{K} \int_{\mathbb{R}} x_{k}(t+\tau) \frac{1}{\sigma(t)} g\left(\frac{\tau}{\sigma(t)}\right) e^{-i 2 \pi \eta \tau} d \tau \\
& =\sum_{k=0}^{K} \int_{\mathbb{R}} x_{k}(t) e^{i 2 \pi\left(\phi_{k}^{\prime}(t) \tau+\frac{1}{2} \phi_{k}^{\prime \prime}(t) \tau^{2}\right)} \frac{1}{\sigma(t)} g\left(\frac{\tau}{\sigma(t)}\right) e^{-i 2 \pi \eta \tau} d \tau+\operatorname{res}_{0} \\
& =\sum_{k=0}^{K} x_{k}(t) G_{k}\left(\sigma(t)\left(\eta-\phi_{k}^{\prime}(t)\right)\right)+\operatorname{res}_{0},
\end{aligned}
$$

where

$$
\operatorname{res}_{0}:=\sum_{k=0}^{K} \int_{\mathbb{R}} x_{k, \mathrm{rl}}(t, \tau) \frac{1}{\sigma(t)} g\left(\frac{\tau}{\sigma(t)}\right) e^{-i 2 \pi \eta \tau} d \tau
$$

Write $x_{k, \mathrm{rl}}(t, \tau)$ as

$$
\begin{aligned}
x_{k, \mathrm{ll}}(t, \tau):= & \left(A_{k}(t+\tau)-A_{k}(t)\right) e^{i 2 \pi \phi_{k}(t+\tau)} \\
& +x_{k}(t) e^{i 2 \pi\left(\phi_{k}^{\prime}(t) \tau+\frac{1}{2} \phi_{k}^{\prime \prime}(t) \tau^{2}\right)}\left(e^{i 2 \pi\left(\phi_{k}(t+\tau)-\phi_{k}(t)-\phi_{k}^{\prime}(t) \tau-\frac{1}{2} \phi_{k}^{\prime \prime}(t) \tau^{2}\right)}-1\right) .
\end{aligned}
$$

Then by 10$)$ and the fact:

$$
\left|e^{i 2 \pi\left(\phi_{k}(t+\tau)-\phi_{k}(t)-\phi_{k}^{\prime}(t) \tau-\frac{1}{2} \phi_{k}^{\prime \prime}(t) \tau^{2}\right)}-1\right| \leq 2 \pi \frac{1}{6} \sup _{\eta \in \mathbb{R}}\left|\phi_{k}^{(3)}(\eta) \tau^{3}\right| \leq \frac{\pi}{3} \varepsilon_{3}|\tau|^{3},
$$

we have

$$
\begin{aligned}
\left|\mathrm{res}_{0}\right| & \leq \sum_{k=0}^{K} \int_{\mathbb{R}} \varepsilon_{1} A_{k}(t)|\tau| \frac{1}{\sigma(t)}\left|g\left(\frac{\tau}{\sigma(t)}\right)\right| d \tau+\sum_{k=0}^{K} A_{k}(t) \int_{\mathbb{R}} \frac{\pi}{3} \varepsilon_{3}|\tau|^{3} \frac{1}{\sigma(t)}\left|g\left(\frac{\tau}{\sigma(t)}\right)\right| d \tau \\
& =\varepsilon_{1} I_{1} \sigma(t) M(t)+\frac{\pi}{3} \varepsilon_{3} I_{3} \sigma^{3}(t) M(t)=: M(t) \Pi_{0}(t)
\end{aligned}
$$

where $I_{n}$ is defined in (16), and

$$
\Pi_{0}(t):=\varepsilon_{1} I_{1} \sigma(t)+\frac{\pi}{3} \varepsilon_{3} I_{3} \sigma^{3}(t)
$$

Thus if $\varepsilon_{1}, \varepsilon_{3}$ are small enough, then $\left|\mathrm{res}_{0}\right|$ is small and hence, $G_{k}\left(\sigma(t)\left(\eta-\phi_{k}^{\prime}(t)\right)\right)$ provides the time-frequency zone for $\widetilde{V}_{x_{k}}(t, \eta)$.

Let $0<\tau_{0}<1$ be a given small number as the threshold for zero. Let $\xi_{k}=\xi_{k}(t)>0$ denote the unique solution of $\left|G_{k}\left(\xi_{k}\right)\right|=\tau_{0}$. (Recall that in this section we assume that $\left|G_{k}(\xi)\right|$ is even and decreasing for $\xi \geq 0$.) Choose $\alpha_{k}=\alpha_{k}(t) \geq \xi_{k}(t)$. Denote

$$
O_{k}=\left\{(t, \eta):\left|\eta-\phi_{k}^{\prime}(t)\right|<\frac{\alpha_{k}}{\sigma(t)}, t \in \mathbb{R}\right\}
$$


Then we have

$$
\left|G_{k}\left(\sigma(t)\left(\eta-\phi_{k}^{\prime}\right)\right)\right| \leq \tau_{0} \text { for any }(t, \eta) \notin O_{k} .
$$

Next we consider, as an example, the case when $g$ is the Gaussian function defined by (19) One can obtain for this $g$ (see e.g. [13, 17]),

$$
G_{k}(\xi)=\frac{1}{\sqrt{1-i 2 \pi \phi_{k}^{\prime \prime}(t) \sigma^{2}(t)}} e^{-\frac{2 \pi^{2} \xi^{2}}{1+\left(2 \pi \phi_{k}^{\prime \prime}(t) \sigma^{2}(t)\right)^{2}}\left(1+i 2 \pi \phi_{k}^{\prime \prime}(t) \sigma^{2}(t)\right)},
$$

where in this paper, $\sqrt{1-i b}, b \in \mathbb{R}$, denotes the complex root of $1-i b$ lying in the same quadrant as $1-i b$. Thus

$$
\left|G_{k}(\xi)\right|=\frac{1}{\left(1+\left(2 \pi \phi_{k}^{\prime \prime}(t) \sigma^{2}(t)\right)^{2}\right)^{\frac{1}{4}}} e^{-\frac{2 \pi^{2}}{1+\left(2 \pi \phi_{k}^{\prime \prime}(t) \sigma^{2}(t)\right)^{2}} \xi^{2}} .
$$

Therefore, in this case, assuming $\tau_{0}\left(1+\left(2 \pi \phi_{k}^{\prime \prime}(t) \sigma^{2}(t)\right)^{2}\right)^{\frac{1}{4}} \leq 1$,

$$
\xi_{k}=\sqrt{1+\left(2 \pi \phi_{k}^{\prime \prime}(t) \sigma^{2}(t)\right)^{2}} \frac{1}{2 \pi} \sqrt{2 \ln \left(\frac{1}{\tau_{0}}\right)-\frac{1}{2} \ln \left(1+\left(2 \pi \phi_{k}^{\prime \prime}(t) \sigma^{2}(t)\right)^{2}\right)} .
$$

As in [17, one may choose

$$
\alpha_{k}=\alpha\left(1+2 \pi\left|\phi_{k}^{\prime \prime}(t)\right| \sigma^{2}(t)\right),
$$

where $\alpha$ is defined by (20). Then $\alpha_{k} \geq \xi_{k}$.

In this section we will assume the multicomponent signal $x(t)$ is well-separated, that is there is $\sigma(t)$ such that

$$
O_{k} \cap O_{\ell}=\emptyset, \quad k \neq \ell .
$$

Refer to [17] for the well-separated condition when $g$ is the Gaussian window function.

From (61) with $\ell=k-1$,

$$
\phi_{k}^{\prime}(t)-\frac{\alpha_{k}}{\sigma(t)} \geq \phi_{k-1}^{\prime}(t)+\frac{\alpha_{k-1}}{\sigma(t)}
$$

or equivalently

$$
\sigma(t)\left(\phi_{k}^{\prime}(t)-\phi_{k-1}^{\prime}(t)\right) \geq \alpha_{k}+\alpha_{k-1} .
$$

More generally, we have for $k \neq \ell$,

$$
\sigma(t)\left|\phi_{k}^{\prime}(t)-\phi_{\ell}^{\prime}(t)\right| \geq \Upsilon_{k, \ell},
$$

where

$$
\Upsilon_{k, \ell}:=\left\{\begin{array}{l}
\alpha_{k}+\alpha_{\ell}+2\left(\alpha_{k-1}+\alpha_{k-2}+\cdots+\alpha_{\ell+1}\right), \text { for } k>\ell \\
\alpha_{k}+\alpha_{\ell}+2\left(\alpha_{\ell-1}+\alpha_{\ell-2}+\cdots+\alpha_{k+1}\right), \text { for } k<\ell
\end{array}\right.
$$


Hence for $(t, \eta) \in O_{\ell}$, the following holds

$$
\sigma(t)\left|\eta-\phi_{k}^{\prime}(t)\right| \geq \sigma(t)\left|\phi_{k}^{\prime}(t)-\phi_{\ell}^{\prime}(t)\right|-\sigma(t)\left|\eta-\phi_{\ell}^{\prime}(t)\right| \geq \Upsilon_{k, \ell}-\alpha_{\ell}
$$

Denote

$$
\begin{aligned}
& \operatorname{Err}_{\ell}=\operatorname{Err}_{\ell}(t):=M(t) \Pi_{0}(t)+\sum_{k \neq \ell} A_{k}(t)\left|G_{k}\left(\Upsilon_{k, \ell}-\alpha_{\ell}\right)\right|, \\
& g_{0}=g_{0}(t):=\min _{0 \leq k \leq K}\left|G_{k}(0)\right|=\min \left\{1,\left|G_{k}(0)\right|, 1 \leq k \leq K\right\},
\end{aligned}
$$

where $\Pi_{0}(t)$ is defined by $(54)$.

Since $\Upsilon_{k, \ell}-\alpha_{\ell} \geq \alpha_{k}$ and $\left|G_{k}\left(\alpha_{k}\right)\right| \leq \tau_{0}$, we have

$$
\operatorname{Err}_{\ell} \leq M(t) \Pi_{0}(t)+\tau_{0} \sum_{k \neq \ell} A_{k}(t) .
$$

For a fixed $t$, and a positive number $\widetilde{\epsilon}_{1}$, we let $\mathcal{G}_{t}$ denote the set defined by $(27)$, and we define

$$
\mathcal{H}_{t, k}:=\left\{\eta \in \mathcal{G}_{t}:\left|\eta-\phi_{k}^{\prime}(t)\right|<\frac{\alpha_{k}}{\sigma(t)}\right\} .
$$

Note that $\mathcal{H}_{t, k}$ depends on $\widetilde{\epsilon}_{1}$, and for simplicity of presentation, we drop $\widetilde{\epsilon}_{1}$ from it. Also observe that $\mathcal{H}_{t, k}=\mathcal{G}_{t} \cap\left\{\eta:(t, \eta) \in O_{k}\right\}$. Denote

$$
\check{\eta}_{0}:=0, \check{\eta}_{\ell}:=\check{\eta}_{\ell}(t):=\operatorname{argmax}_{\eta \in \mathcal{H}_{t, \ell}}\left|\widetilde{V}_{x}(t, \eta)\right|, \quad \ell=1, \cdots, K \text {. }
$$

Let $\mathcal{D}_{\varepsilon_{1}, \varepsilon_{3}}$ denote the set of multicomponent signals of (5) satisfying (8)-(10), 45), 46.

Theorem 2. Let $x(t) \in \mathcal{D}_{\varepsilon_{1}, \varepsilon_{3}}$ for some small $\varepsilon_{1}, \varepsilon_{3}>0$ and $g$ be an admission window function. Suppose for this $x(t)$, there is a function $\sigma(t)>0$ such that 61) holds, and $\varepsilon_{1}, \varepsilon_{3}, \tau_{0}$ are small enough such that

$$
\operatorname{Err}_{\ell}<\frac{1}{2}\left|G_{\ell}(0)\right| A_{\ell}(t), 2 M(t)\left(\tau_{0}+\Pi_{0}(t)\right) \leq g_{0}(t) \mu(t) .
$$

Let $\widetilde{\epsilon}_{1}$ be a function satisfying

$$
M(t)\left(\tau_{0}+\Pi_{0}(t)\right) \leq \widetilde{\epsilon}_{1} \leq g_{0}(t) \mu(t)-M(t)\left(\tau_{0}+\Pi_{0}(t)\right) .
$$

Then the following statements hold.

(a) Let $\mathcal{G}_{t}$ and $\mathcal{H}_{t, k}$ be the sets defined by (27) and (65) respectively for some $\widetilde{\epsilon}_{1}$ satisfying (67). Then $\mathcal{G}_{t}$ can be expressed as a disjoint union of exactly $K+1$ non-empty sets $\mathcal{H}_{t, k}, 0 \leq k \leq K$.

(b) Let $\check{\eta}_{\ell}$ be defined by (66). Then for $\ell=1, \cdots, K$,

$$
\left|\check{\eta}_{\ell}(t)-\phi_{\ell}^{\prime}(t)\right| \leq \mathrm{Bd}_{1, \ell}:=\frac{1}{\sigma(t)}\left|G_{\ell}\right|^{-1}\left(\left|G_{\ell}(0)\right|-\frac{2 \operatorname{Err}_{\ell}(t)}{A_{\ell}(t)}\right)
$$

where $\mathrm{Err}_{\ell}$ is defined by 63 . 
(c) For $\ell=0, \cdots, K$,

$$
\left|\widetilde{V}_{x}\left(t, \check{\eta}_{\ell}\right)-G_{\ell}(0) x_{\ell}(t)\right| \leq \operatorname{Bd}_{2, \ell}:=\operatorname{Err}_{\ell}(t)+2 \pi I_{1} A_{\ell}(t)\left|\widehat{G}_{\ell}\right|^{-1}\left(\left|G_{\ell}(0)\right|-\frac{2 \operatorname{Err}_{\ell}(t)}{A_{\ell}(t)}\right) .
$$

The proof of Theorem 2 will be deferred to the end of this section.

From Theorem 2, we have that $\check{\eta}_{\ell}$ defined by 66 provides an approximation to $\phi_{\ell}^{\prime}(t)$. In addition, we have the recovery formula

$$
x_{\ell}(t) \approx \frac{1}{G_{\ell}(0)} \widetilde{V}_{x}\left(t, \check{\eta}_{\ell}\right)
$$

For a real $x(t)$, the recovery formula will be

$$
x_{\ell}(t) \approx 2 \operatorname{Re}\left(\frac{1}{G_{\ell}(0)} \widetilde{V}_{x}\left(t, \check{\eta}_{\ell}\right)\right)
$$

In some case $\check{\eta}_{\ell}$ is $\widehat{\eta}_{\ell}$ defined by (28). Thus in this case, the linear chirp-based model does not provide a more accurate IF approximation than the sinusoidal signal-based model. However, the component recovery formula derived from linear chirp local approximation has a factor $\frac{1}{G_{\ell}(0)}$ as shown in (70), which distinguishes the linear chirp-based model from the sinusoidal signal-based model.

Recall that when $g(t)$ is the Gaussian window function given by (19), then the corresponding $G_{k}(\xi)$ defined by $(48)$ and $\left|G_{k}(\xi)\right|$ is given by (58). Observe that if we choose $\alpha_{k}$ as the quantity given by 60 , then

$$
\left|G_{k}\left(\Upsilon_{k, \ell}-\alpha_{\ell}\right)\right| \leq\left|G_{k}\left(\alpha_{k}\right)\right| \leq \frac{1}{\left(1+\left(2 \pi \phi_{k}^{\prime \prime}(t) \sigma^{2}(t)\right)^{2}\right)^{\frac{1}{4}}} e^{-2 \pi^{2} \alpha^{2}} \leq e^{-2 \pi^{2} \alpha^{2}} .
$$

Hence the terms $\left|G_{k}\left(\Upsilon_{k, \ell}-\alpha_{\ell}\right)\right|$ in $\operatorname{Err}_{\ell}$ defined by (63) are very small if $\alpha$ is quite large, say $\alpha \geq 1$. In addition, we have

$$
\left|G_{\ell}\right|^{-1}(\xi)=\frac{1}{\pi \sqrt{2}\left|G_{\ell}(0)\right|^{2}}\left(-\ln \frac{\xi}{\left|G_{\ell}(0)\right|}\right)^{1 / 2}, 0<\xi<\left|G_{\ell}(0)\right|
$$

where

$$
\left|G_{\ell}(0)\right|=\left(1+\left(2 \pi \phi_{\ell}^{\prime \prime}(t) \sigma^{2}(t)\right)^{2}\right)^{-\frac{1}{4}} .
$$

Hence the error bound $\mathrm{Bd}_{1, \ell}$ in $(68)$ is

$$
\mathrm{Bd}_{1, \ell}=\frac{1}{\sigma(t)}\left|G_{\ell}\right|^{-1}\left(\left|G_{\ell}(0)\right|-\frac{2 \operatorname{Err}_{\ell}(t)}{A_{\ell}(t)}\right)=\frac{1}{\sigma(t) \pi \sqrt{2}\left|G_{\ell}(0)\right|^{2}}\left(-\ln \left(1-\frac{2 \operatorname{Err}_{\ell}(t)}{\left|G_{\ell}(0)\right| A_{\ell}(t)}\right)^{1 / 2} .\right.
$$


Suppose $\varepsilon_{1}, \varepsilon_{3}, \tau_{0}$ are small enough such that $\operatorname{Err}_{\ell}(t) \leq \frac{1}{4}\left|G_{\ell}(0)\right| A_{\ell}(t)$. Then applying the fact $-\ln (1-t)<2 t$ for $0<t<\frac{1}{2}$ again, we have

$$
\operatorname{Bd}_{1, \ell}<\frac{\sqrt{2}}{\sigma(t) \pi} \frac{1}{\left|G_{\ell}(0)\right|^{5 / 2}} \sqrt{\frac{\operatorname{Err}_{\ell}(t)}{A_{\ell}(t)}} .
$$

Also, in this case, we have

$$
\operatorname{Bd}_{2, \ell}<\operatorname{Err}_{\ell}(t)+\frac{2 \sqrt{2} I_{2}}{\left|G_{\ell}(0)\right|^{5 / 2}} \sqrt{A_{\ell}(t) \operatorname{Err}_{\ell}(t)}
$$

To summarize, we have the following corollary.

Corollary 3. Let $x(t) \in \mathcal{D}_{\varepsilon_{1}, \varepsilon_{2}}$ for some $\varepsilon_{1}, \varepsilon_{2}>0$. Suppose the conditions in Theorem 2 for the Gaussian window function $g$ given by (19) are satisfied and that $\operatorname{Err}_{\ell}(t)<\frac{1}{4}\left|G_{\ell}(0)\right| A_{\ell}(t)$. Then Part (a) of Theorem 2 holds; and with $\check{\eta}_{\ell}$ defined by (66), we have

$$
\begin{aligned}
& \left|\phi_{\ell}^{\prime}(t)-\check{\eta}_{\ell}(t)\right|<\frac{\sqrt{2}}{\sigma(t) \pi}\left(1+\left(2 \pi \phi_{\ell}^{\prime \prime}(t) \sigma^{2}(t)\right)^{2}\right)^{\frac{5}{8}} \sqrt{\frac{\operatorname{Err}_{\ell}(t)}{A_{\ell}(t)}}, \quad \ell=1,2, \cdots, K, \\
& \left|x_{\ell}(t)-\sqrt{1-i 2 \pi \phi_{\ell}^{\prime \prime}(t) \sigma^{2}(t)} \widetilde{V}_{x}\left(t, \check{\eta}_{\ell}\right)\right|<\left(1+\left(2 \pi \phi_{\ell}^{\prime \prime}(t) \sigma^{2}(t)\right)^{2}\right)^{\frac{1}{4}} \operatorname{Err}_{\ell}(t) \\
& +2 \sqrt{2} I_{1}\left(1+\left(2 \pi \phi_{\ell}^{\prime \prime}(t) \sigma^{2}(t)\right)^{2}\right)^{\frac{7}{8}} \sqrt{A_{\ell}(t) \operatorname{Err}_{\ell}(t)}, \quad \ell=0,1, \cdots, K,
\end{aligned}
$$

where $\operatorname{Err}_{\ell}(t)$ is defined by (63).

Remark 3. From (73), we will use the following recovery formula for a real $x(t)$ :

$$
x_{\ell}(t) \approx 2 \operatorname{Re}\left(\sqrt{1-i 2 \pi \phi_{\ell}^{\prime \prime}(t) \sigma^{2}(t)} \widetilde{V}_{x}\left(t, \check{\eta}_{\ell}\right)\right) .
$$

(74) was first derived in [19] using linear chirp local approximation. Here we provide an error bound as shown in 73 .

Remark 4. Observe that a smaller $\sigma(t)$ results in smaller error bounds in (73) for component recovery. Note that the IF estimate error bound in 72 is

$$
\frac{\sqrt{2}}{\pi \sqrt{A_{\ell}(t)}}\left(1+\left(2 \pi \phi_{\ell}^{\prime \prime}(t) \sigma^{2}(t)\right)^{2}\right)^{\frac{5}{8}}\left(\frac{M(t)}{\sigma(t)} \varepsilon_{1} I_{1}+M(t) \frac{\pi}{3} \varepsilon_{3} I_{3} \sigma(t)+\frac{1}{\sigma^{2}(t)} \sum_{k \neq \ell} A_{k}(t)\left|G_{k}\left(\Upsilon_{k, \ell}-\alpha_{\ell}\right)\right|\right)^{1 / 2} .
$$

Thus for IF estimate, if $\phi_{\ell}^{\prime \prime}(t)$ is quite large, then we should choose a smaller $\sigma(t)$, which should not be too small to avoid a possible large error caused by $\frac{M(t)}{\sigma(t)} \varepsilon_{1} I_{1}$ or $/$ and $\frac{1}{\sigma^{2}(t)} \sum_{k \neq \ell} A_{k}(t) \mid G_{k}\left(\Upsilon_{k, \ell}-\right.$ $\left.\alpha_{\ell}\right) \mid$.

Remark 5. Unlike Theorem 1 and Corollaries 1 and 2, there is no restriction of the amplitude of $\phi_{k}^{\prime \prime}(t)$ for the source signal $x(t)$ to be analyzed in Theorem 2 and Corollary 3 . However, as we see from (68) (69) and (72) (73), $\phi_{k}^{\prime \prime}(t)$ still effects the IF estimate error and component recovery error. 
Next we provide the proof of Theorem 2 , which is similar to that of Theorem 1 .

Proof of Theorem 2(a). Clearly $\cup_{k=0}^{K} \mathcal{H}_{t, k} \subseteq \mathcal{G}_{t}$. Next we show $\mathcal{G}_{t} \subseteq \cup_{k=0}^{K} \mathcal{H}_{t, k}$. Let $\eta \in \mathcal{G}_{t}$. Assume $\eta \notin \cup_{k=0}^{K} \mathcal{H}_{t, k}$. That is $(t, \eta) \notin \cup_{k=0}^{K} O_{k}$. From (56), we have $\left|G_{k}\left(\sigma(t)\left(\eta-\phi_{k}^{\prime}(t)\right)\right)\right| \leq \tau_{0}$ for any $k$. Hence, by (51) and (53), we have

$$
\begin{aligned}
\left|\widetilde{V}_{x}(t, \eta)\right| & \leq \sum_{k=0}^{K}\left|x_{k}(t) G_{k}\left(\sigma(t)\left(\eta-\phi_{k}^{\prime}(t)\right)\right)\right|+\left|\operatorname{res}_{0}\right| \\
& \leq \tau_{0} \sum_{k=0}^{K} A_{k}(t)+M(t) \Pi_{0}(t) \leq \widetilde{\epsilon}_{1}
\end{aligned}
$$

a contradiction to the assumption $\left|\widetilde{V}_{x}(t, \eta)\right|>\widetilde{\epsilon}_{1}$. Thus $(t, \eta) \in O_{\ell}$ for some $\ell$, and hence $\eta \in \mathcal{H}_{t, \ell}$. This shows $\mathcal{G}_{t}=\cup_{k=0}^{K} \mathcal{H}_{t, k}$. Since $O_{k}, 0 \leq k \leq K$ are not overlapping, we know $\mathcal{H}_{t, k}, 0 \leq k \leq K$ are disjoint.

To show $\mathcal{H}_{t, \ell}$ is non-empty, we need only to show $\phi_{\ell}^{\prime}(t) \in \mathcal{G}_{t}$ which implies $\phi_{\ell}^{\prime}(t) \in \mathcal{H}_{\ell, t}$. From

$$
\begin{aligned}
\left|\widetilde{V}_{x}\left(t, \phi_{\ell}^{\prime}(t)\right)\right| & \geq \sum_{k=0}^{K}\left|x_{k}(t) G_{k}\left(\sigma(t)\left(\phi_{\ell}^{\prime}(t)-\phi_{k}^{\prime}(t)\right)\right)\right|-\left|\operatorname{res}_{0}\right| \\
& \geq\left|x_{\ell}(t) G_{\ell}(0)\right|-\sum_{k \neq \ell}\left|x_{k}(t)\right| \tau_{0}-M(t) \Pi_{0}(t) \\
& \geq\left|G_{\ell}(0)\right| A_{\ell}(t)-M(t) \tau_{0}-M(t) \Pi_{0}(t) \\
& \geq g_{0}(t) \mu(t)-M(t)\left(\tau_{0}+\Pi_{0}(t)\right) \geq \widetilde{\epsilon}_{1},
\end{aligned}
$$

we conclude that $\phi_{\ell}^{\prime}(t) \in \mathcal{G}_{t}$. Therefore, the statements in (a) hold.

Proof of Theorem $2(\mathbf{b})$. For any $\eta \in \mathcal{H}_{t, \ell}$, from (51), we have

$$
\begin{aligned}
& \mid \widetilde{V}_{x}(t, \eta)-x_{\ell}(t) G_{\ell}\left(\sigma(t)\left(\eta-\phi_{\ell}^{\prime}(t)\right) \mid\right. \\
& =\mid \sum_{k \neq \ell} x_{k}(t) G_{k}\left(\sigma(t)\left(\eta-\phi_{k}^{\prime}(t)\right)+\operatorname{res}_{0} \mid\right. \\
& \leq \sum_{k \neq \ell} A_{k}(t)\left|G_{k}\left(\Upsilon_{k, \ell}-\alpha_{\ell}\right)\right|+M(t) \Pi_{0}(t),
\end{aligned}
$$

where the last inequality follows from (53), (62) and the assumption that $\left|G_{k}(\xi)\right|$ is decreasing for $\xi \geq 0$. Thus

$$
\mid \widetilde{V}_{x}(t, \eta)-x_{\ell}(t) G_{\ell}\left(\sigma(t)\left(\eta-\phi_{\ell}^{\prime}(t)\right) \mid \leq \operatorname{Err}_{\ell}(t) .\right.
$$

Hence, letting $\eta=\phi_{\ell}^{\prime}(t)$, we have

$$
\left|\widetilde{V}_{x}\left(t, \phi_{\ell}^{\prime}(t)\right)\right| \geq\left|x_{\ell}(t) G_{\ell}(0)\right|-\operatorname{Err}_{\ell}(t)=\left|G_{\ell}(0)\right| A_{\ell}(t)-\operatorname{Err}_{\ell}(t)
$$


By the definition of $\check{\eta}_{\ell}$ and 75$)$ again, we have

$$
\left|\widetilde{V}_{x}\left(t, \phi_{\ell}^{\prime}(t)\right)\right| \leq\left|\widetilde{V}_{x}\left(t, \breve{\eta}_{\ell}\right)\right| \leq\left|x_{\ell}(t) G_{\ell}\left(\sigma(t)\left(\check{\eta}_{\ell}-\phi_{\ell}^{\prime}(t)\right)\right)\right|+\operatorname{Err}_{\ell}(t)
$$

This, together with (76), implies

$$
\left|G_{\ell}(0)\right| A_{\ell}(t)-\operatorname{Err}_{\ell}(t) \leq A_{\ell}(t)\left|G_{\ell}\left(\sigma(t)\left(\check{\eta}_{\ell}-\phi_{\ell}^{\prime}(t)\right)\right)\right|+\operatorname{Err}_{\ell}(t),
$$

or equivalently

$$
\left|G_{\ell}(0)\right|-\frac{2 \operatorname{Err}_{\ell}(t)}{A_{\ell}(t)} \leq\left|G_{\ell}\left(\sigma(t)\left(\check{\eta}_{\ell}-\phi_{\ell}^{\prime}(t)\right)\right)\right| .
$$

Since $\left|G_{\ell}(\xi)\right|$ is decreasing for $\xi \geq 0$, we have

$$
\sigma(t)\left|\check{\eta}_{\ell}-\phi_{\ell}^{\prime}(t)\right| \leq\left|G_{\ell}\right|^{-1}\left(\left|G_{\ell}(0)\right|-\frac{2 \operatorname{Err}_{\ell}(t)}{A_{\ell}(t)}\right) .
$$

Thus shows 68.

Proof of Theorem 2(c). From (75), we have

$$
\begin{aligned}
& \left|\widetilde{V}_{x}\left(t, \check{\eta}_{\ell}\right)-G_{\ell}(0) x_{\ell}(t)\right| \\
& \leq\left|\widetilde{V}_{x}\left(t, \check{\eta}_{\ell}\right)-x_{\ell}(t) G_{\ell}\left(\sigma(t)\left(\check{\eta}_{\ell}-\phi_{\ell}^{\prime}(t)\right)\right)\right|+\left|x_{\ell}(t) G_{\ell}\left(\sigma(t)\left(\check{\eta}_{\ell}-\phi_{\ell}^{\prime}(t)\right)\right)-G_{\ell}(0) x_{\ell}(t)\right| \\
& \leq \operatorname{Err}_{\ell}(t)+A_{\ell}(t)\left|\int_{\mathbb{R}} g(\tau)\left(e^{i \pi \sigma^{2}(t) \phi_{\ell}^{\prime \prime}(t) \tau^{2}} e^{-i 2 \pi \sigma(t)\left(\check{\eta}_{\ell}-\phi_{\ell}^{\prime}(t)\right) \tau}-e^{i \pi \sigma^{2}(t) \phi_{\ell}^{\prime \prime}(t) \tau^{2}}\right) d \tau\right| \\
& \leq \operatorname{Err}_{\ell}(t)+A_{\ell}(t) \int_{\mathbb{R}}|g(\tau)| 2 \pi \sigma(t)\left|\check{\eta}_{\ell}-\phi_{\ell}^{\prime}(t)\right||\tau| d \tau \\
& \leq \operatorname{Err}_{\ell}(t)+A_{\ell}(t) 2 \pi\left|G_{\ell}\right|^{-1}\left(\left|G_{\ell}(0)\right|-\frac{2 \operatorname{Err}_{\ell}(t)}{A_{\ell}(t)}\right) \int_{\mathbb{R}}|g(\tau)||\tau| d \tau \\
& =\operatorname{Err}_{\ell}(t)+2 \pi I_{1} A_{\ell}(t)\left|G_{\ell}\right|^{-1}\left(\left|G_{\ell}(0)\right|-\frac{2 \operatorname{Err}_{\ell}(t)}{A_{\ell}(t)}\right) .
\end{aligned}
$$

This completes the proof of 69).

\section{Experiments}

In this section we provide some experimental results. We focus on the comparison between the performances of sinusoidal signal-based and linear chirp-based models in component recovery. The readers are referred to [19] for experiments which compare our linear chirp-based model with SST and the 2nd-order SST in component recovery. In the first two examples we consider monocomponent signals, where we simple let $\sigma=\frac{1}{16}$. In all our experiments, we use the Gaussian function $g(t)$ defined by 19 as the window function. The signals considered in our experiments 
are uniformly sampled. Suppose $t_{m}=m \triangle t, m=0,1, \cdots, N-1$ be the sample points. Then finite sequence $\left(\widehat{\eta}_{\ell}\left(t_{m}\right)\right)_{0 \leq m<N}$ obtained by (28) or $\left(\breve{\eta}_{\ell}\left(t_{m}\right)\right)_{0 \leq m<N}$ in (66) is an estimate to IF $\phi_{\ell}^{\prime}\left(t_{m}\right)$. In our experiments $\widehat{\eta}_{\ell}\left(t_{m}\right)=\check{\eta}_{\ell}\left(t_{m}\right)$. When we use an linear chirp-based model recovery formula such as (71) or (74), we need to estimate $\phi_{\ell}^{\prime \prime}(t)$. [19] provides a method to estimate $\phi_{\ell}^{\prime \prime}(t)$. Here we use a five-point formula (see, e.g. [3]) to obtain an approximation to $\phi_{\ell}^{\prime \prime}\left(t_{m}\right)$. More precisely, we first smooth $\check{\eta}_{\ell}\left(t_{m}\right), 0 \leq m<N$ by a filter (here we use cubic B-spline filter $\left\{\frac{1}{16}, \frac{4}{16} \frac{6}{16} \frac{4}{16} \frac{1}{16}\right\}$ ). After that we apply a five-point formula to the smoothed $\check{\eta}_{\ell}\left(t_{m}\right), 0 \leq m<N$ to obtain an approximation to $\left(\phi_{\ell}^{\prime \prime}\left(t_{m}\right)\right)_{0 \leq m<N}$, denoted as $\left(r_{\ell}\left(t_{m}\right)\right)_{0 \leq m<N}$. Finally this approximation is smoothed by the cubic B-spline filter and the resulting sequence, denoted as $\left(\widetilde{r}_{\ell}\left(t_{m}\right)\right)_{0 \leq m<N}$, is used in (74) as $\phi_{\ell}^{\prime \prime}(t)$. In the following examples, in order to avoid the boundary effect, we provide the recovery errors over $[N / 8+1: 7 N / 8]$ in either figures or a table below.
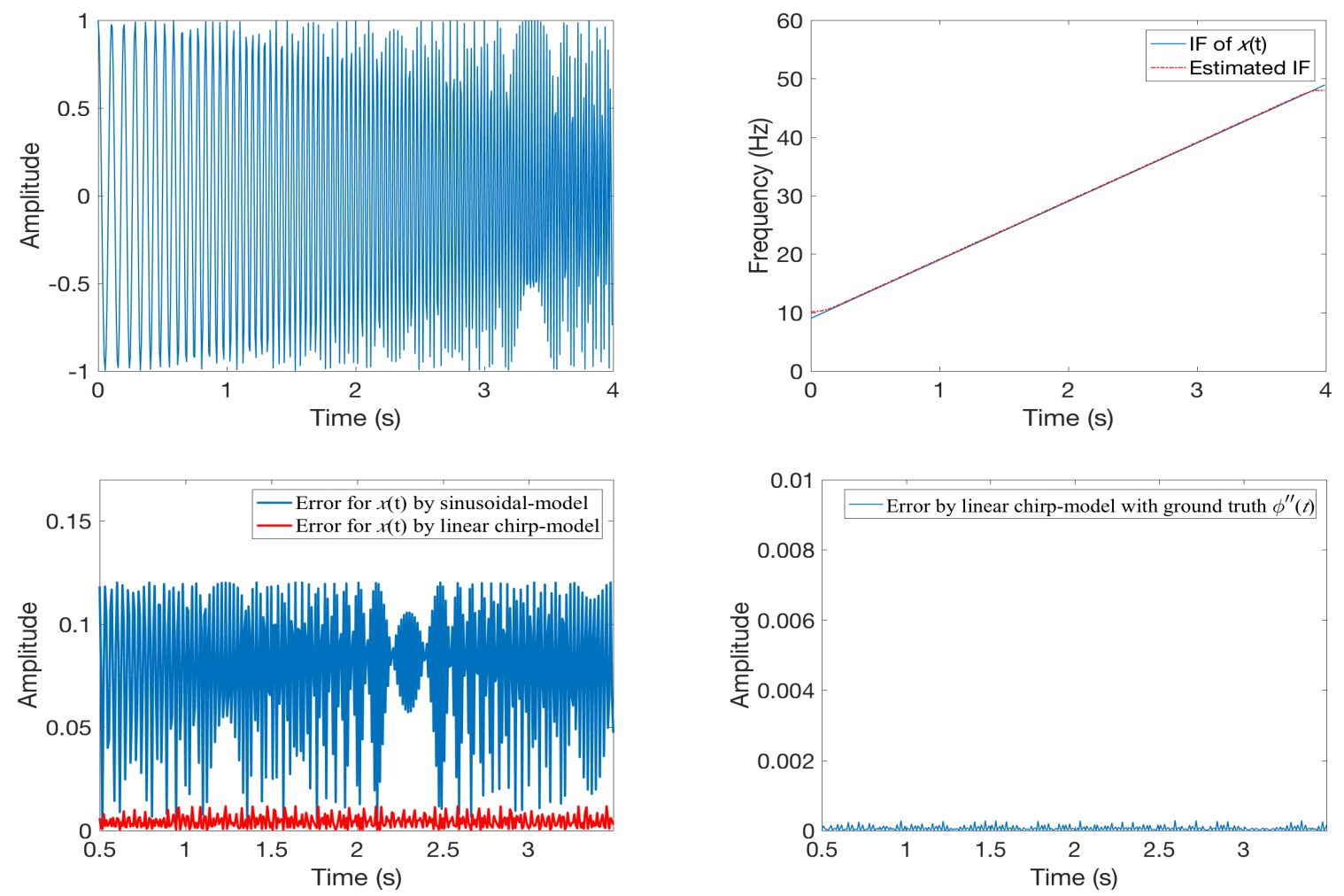

Figure 1: Example of monocomponent signal $x(t)$ in 777 . Top-left: Waveform $x(t)$; Top-right: IF $\phi^{\prime}(t)$ of $x(t)$ (solid line) and estimated IF $\widehat{\eta}(t)$ (red dot-dashed line); Bottom-left: Absolute recovery errors for $x(t)$ by sinusoidal signal-based model (blue line) and by linear chirp-based model with estimated $\phi^{\prime \prime}(t)$ (red line); Bottom-right: Absolute recovery error for $x(t)$ by linear chirp-based model with ground truth $\phi^{\prime \prime}(t)$.

Let $x(t)$ be a linear chirp given by

$$
x(t)=\cos \left(2 \pi\left(9 t+5 t^{2}\right)\right), \quad t \in[0,4),
$$



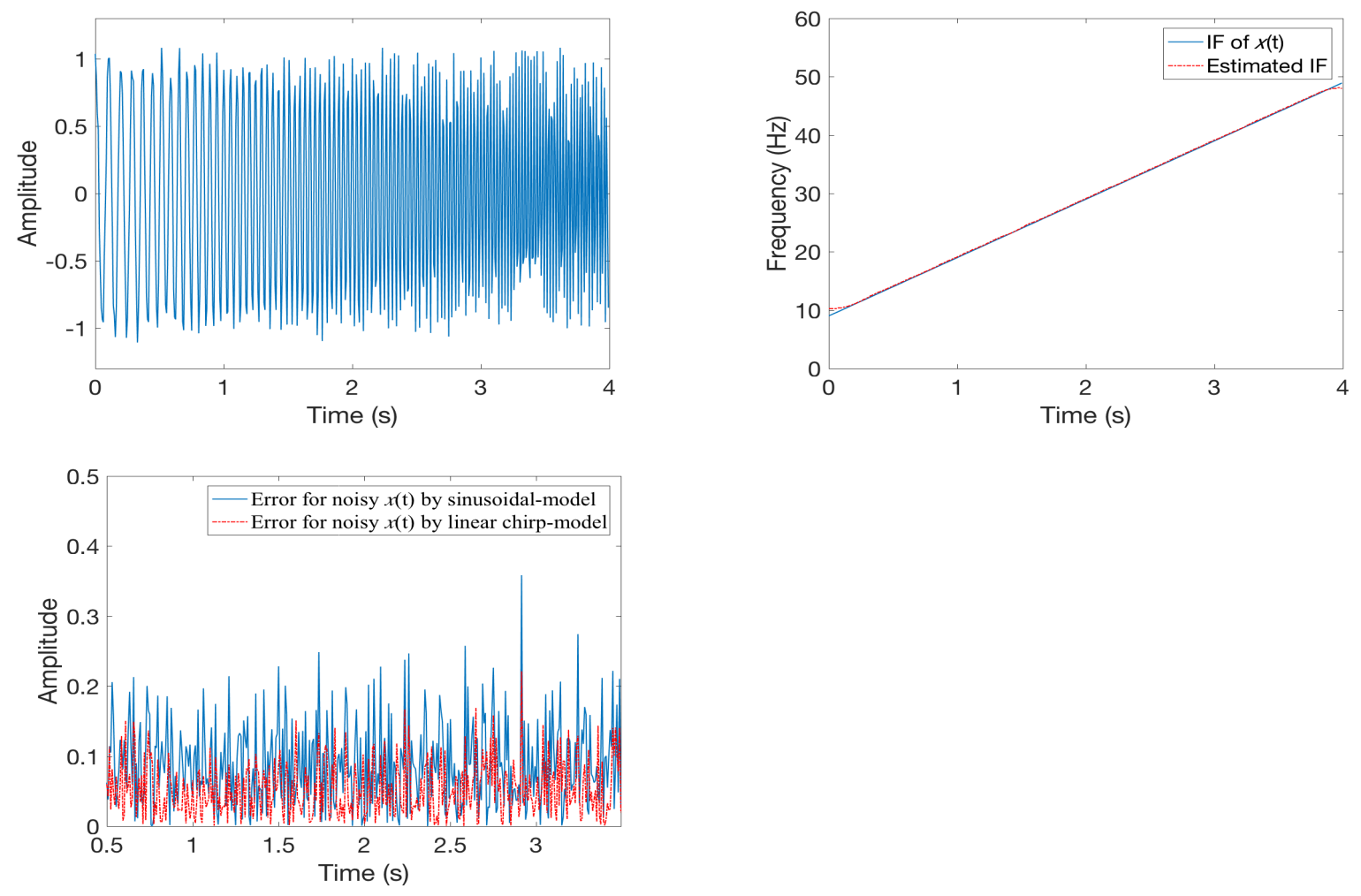

Figure 2: Example of signal $x(t)$ in 477 contaminated by a noise with 10dB. Top-left: Noisy $x(t)$; Topright: IF $\phi^{\prime}(t)$ of $x(t)$ (solid line) and estimated IF $\widehat{\eta}(t)$ of noisy $x(t)$ (red dot-dashed line); Bottom: Absolute recovery errors for noisy $x(t)$ by sinusoidal signal-based model (blue line) and by linear chirp-based model with an estimated $\phi^{\prime \prime}(t)$ (red dot-dashed line).

where the number of sampling points is $N=512$ and the sampling rate is $128 \mathrm{~Hz}$. The IF of $x(t)$ is $\phi^{\prime}(t)=9+10 t$, and the chirp rate of $x(t)$ is $\phi^{\prime \prime}(t)=10$. In the top row of Fig 1 , we show the waveform of $x(t)$ and IF $\phi^{\prime}(t)$. In this example and next example, we simply let $\sigma(t) \equiv \sigma=\frac{1}{16}$. The estimated IF, $\left(\widehat{\eta}\left(t_{m}\right)\right)_{0 \leq m<N}\left(\right.$ where $\left.\widehat{\eta}\left(t_{m}\right)=\breve{\eta}\left(t_{m}\right)\right)$, is shown on the top-right panel in Fig. 1 as a red dot-dashed line. The absolute recovery errors

$$
\left|x\left(t_{m}\right)-2 \operatorname{Re}\left(\widetilde{V}_{x}\left(t_{m}, \widehat{\eta}\left(t_{m}\right)\right)\right)\right| \text { and }\left|x\left(t_{m}\right)-2 \operatorname{Re}\left(\sqrt{1-i 2 \pi \widetilde{r}\left(t_{m}\right) \sigma^{2}} \widetilde{V}_{x}\left(t_{m}, \check{\eta}\left(t_{m}\right)\right)\right)\right|
$$

by sinusoidal signal-based model and by linear chirp-based model with $\widetilde{r}\left(t_{m}\right)$ as an approximation to $\phi^{\prime \prime}(t)$ by five-point formula are shown on the bottom-left panel in Fig 1 as a blue line and a red line respectively. Clearly, the recovery error by linear chirp-based model is much smaller. In the bottom-right panel in Fig 1, we provide recovery error $\left|x\left(t_{m}\right)-2 \operatorname{Re}\left(\sqrt{1-i 2 \pi \phi^{\prime \prime}\left(t_{m}\right) \sigma^{2}} \widetilde{V}_{x}\left(t_{m}, \grave{\eta}\left(t_{m}\right)\right)\right)\right|$. We know in this case, the error is very small.

We also consider the performance of models in noisy environment. The results are provided in Fig,2. Again, linear chirp-based model performs better than sinusoidal signal-based model. 

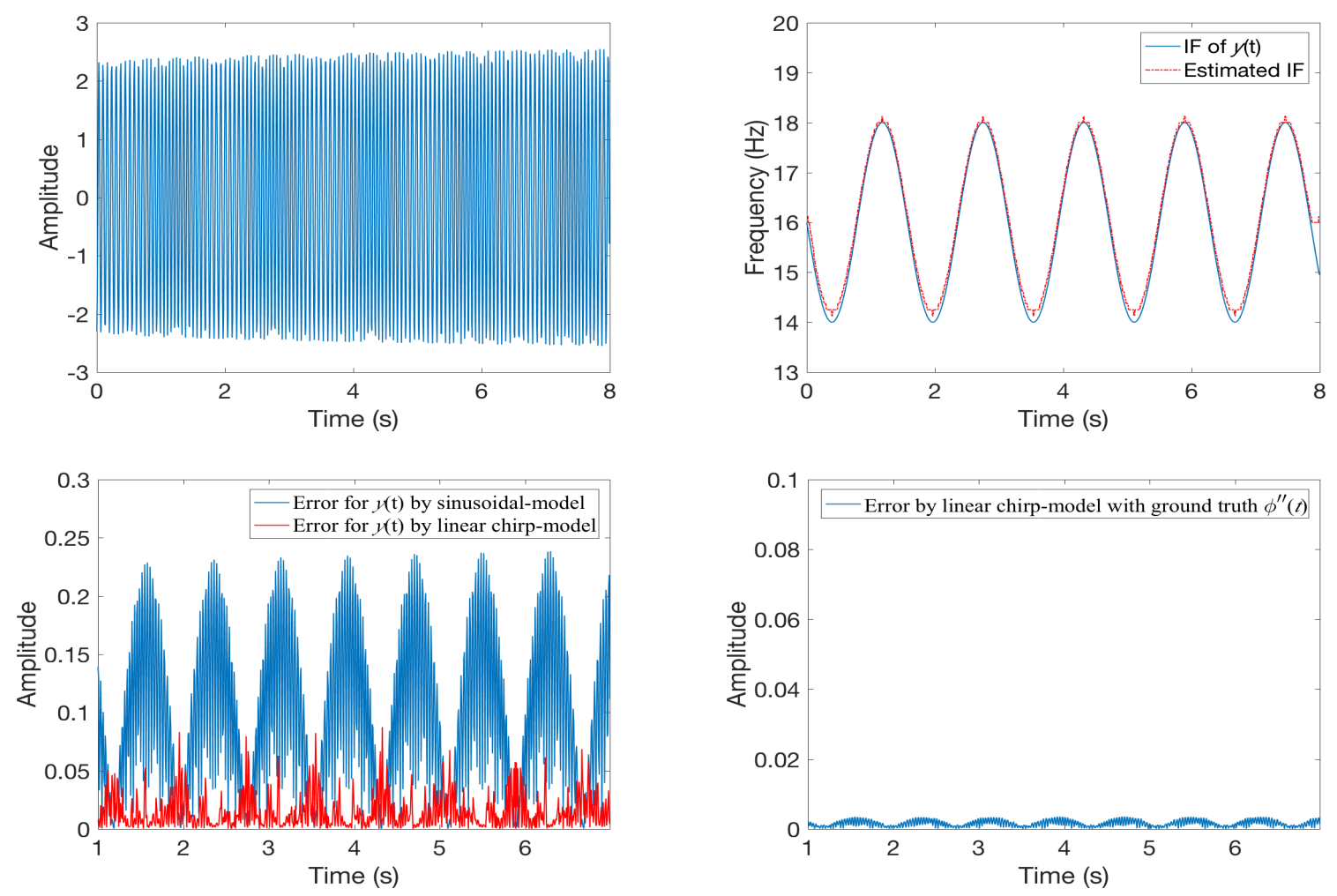

Figure 3: Example of monocomponent signal $y(t)$ in (78). Top-left: Waveform $y(t)$; Top-right: IF $\phi^{\prime}(t)$ of $y(t)$ (solid line) and estimated IF $\widehat{\eta}(t)$ (red dot-dashed line); Bottom-left: Absolute recovery errors for $y(t)$ by sinusoidal signal-based model (blue line) and by linear chirp-based model with estimated $\phi^{\prime \prime}(t)$ (red line); Bottom-right: Absolute recovery error for $y(t)$ by linear chirp-based model with ground truth $\phi^{\prime \prime}(t)$.

Next we consider another type of signal given by

$$
y(t)=\ln (10+\sqrt{t}) \cos (2 \pi(16 t+0.5 \cos (4 t))), \quad t \in[0,8) .
$$

This time IF of $y(t)$ is a sinusoidal function. $y(t)$ is uniformly sampled with the number of sampling points $N=1024$ and the sampling rate $128 \mathrm{~Hz}$. The waveform of $y(t)$ and its IF $\phi^{\prime}(t)$ are shown in the top row of Fig 3 , where the estimated IF $\left(\widehat{\eta}\left(t_{m}\right)\right)_{0 \leq m<N}$ is also provided (on the top-right panel) as a red dot-dashed line. The absolute recovery errors by sinusoidal signal-based model and by linear chirp-based model with $\widetilde{r}\left(t_{m}\right)$ as an approximation to $\phi^{\prime \prime}(t)$ by five-point formula are shown on the bottom-left panel in Fig 3 as a blue line and a red line respectively. By the way, we also provide the recovery error by linear chirp-based model with ground truth $\phi^{\prime \prime}(t)$. In addition, we also provide recovery errors for noisy $y(t)$ in Fig 4 . Again, linear chirp-based model results in smaller recovery errors. 

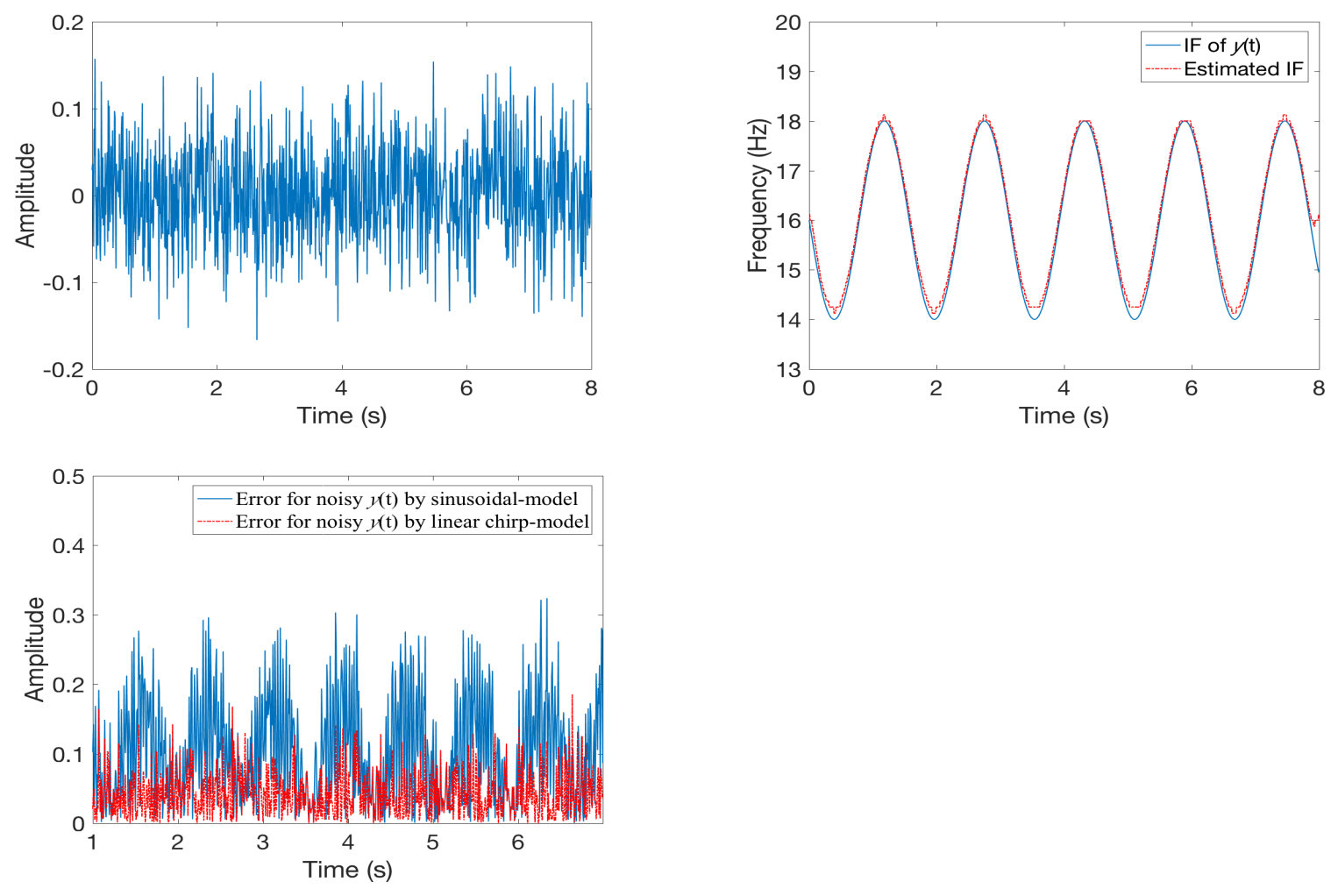

Figure 4: Example of signal $y(t)$ in 78 contaminated by a noise with 15dB. Top-left: Added noise; Top-right: IF $\phi^{\prime}(t)$ of $y(t)$ (solid line) and estimated IF $\widehat{\eta}(t)$ of noisy $y(t)$ (red dot-dashed line); Bottom: Absolute recovery errors for noisy $y(t)$ by sinusoidal signal-based model (blue line) and by linear chirp-based model with an estimated $\phi^{\prime \prime}(t)$ (red dot-dashed line).

Finally, we consider a two-component signal given by

$$
z(t)=z_{1}(t)+z_{2}(t)=\cos \left(2 \pi\left(10 t+5 t^{2}\right)\right)+\cos \left(2 \pi\left(20 t+9 t^{2}\right)\right), \quad t \in[0,1),
$$

where $z(t)$ is uniformly sampled with the number of sampling points $N=128$ and the sampling rate $128 \mathrm{~Hz}$. The waveform of $z(t)$ and its IFs $\phi_{1}^{\prime}(t), \phi_{2}^{\prime}(t)$ are shown in the top row of Fig.5. In this example, we also use a time-varying parameter $\sigma(t)$. The authors in [17] propose an algorithm to select $\sigma(t)$. Here we let $\sigma(t)=\sigma_{2}(t)$ suggested in [17]. $\sigma_{2}(t)$ is shown in the second row of Fig.5. With this $\sigma_{2}(t)$, the estimated IFs $\left(\widehat{\eta}_{\ell}\left(t_{m}\right)\right)_{0 \leq m<N}, \ell=1,2$ are provided on the top-right panel of Fig 5 as red dot-dashed lines. The absolute recovery errors for $z_{1}(t)$ by sinusoidal signal-based model and by linear chirp-based model with $\widetilde{r}_{1}\left(t_{m}\right)$ as an approximation to $\phi_{1}^{\prime \prime}(t)$ by five-point formula are shown on the bottom-left panel of Fig 5 as a blue line and a red line respectively, while the recovery errors for $z_{2}(t)$ are presented on the bottom-right panel of Fig 5 . Linear chirp-based model clearly separates the components $z_{1}(t), z_{2}(t)$ of $z(t)$ better than sinusoidal signal-based model. 

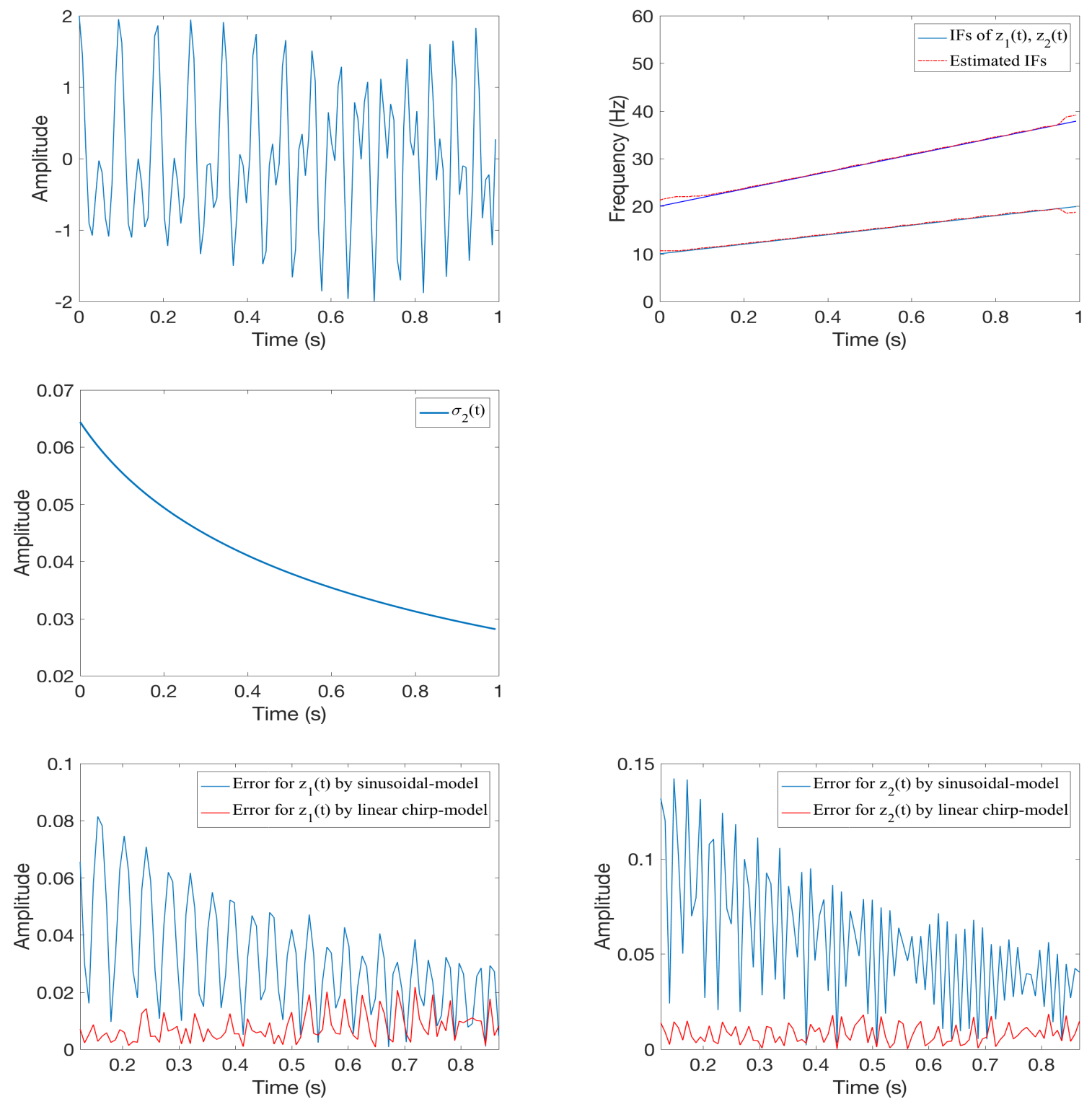

Figure 5: Example of monocomponent signal $z(t)$ in $(79)$. Top-left: Waveform $Z(t)$; Top-right: IFs $\phi_{1}^{\prime}(t), \phi_{2}^{\prime}(t)$ of $z_{1}(t), z_{2}(t)$ (solid line) and estimated IFs $\widehat{\eta}_{1}(t), \widehat{\eta}_{2}(t)$ (red dot-dashed line); Middle: Timevarying parameter $\sigma_{2}(t)$; Bottom-left: Absolute recovery errors for $z_{1}(t)$ by sinusoidal signal-based model (blue line) and by linear chirp-based model with estimated $\phi_{1}^{\prime \prime}(t)$ (red line); Absolute recovery errors for $z_{2}(t)$ by sinusoidal signal-based model (blue line) and by linear chirp-based model with estimated $\phi_{2}^{\prime \prime}(t)$ (red line). 


\begin{tabular}{|l|c|c|c|}
\hline & ASTFT $_{\mathrm{si}}$ & ASTFT $_{\text {lc }}$ & ASTFT $_{\text {lc }}$ with $\phi^{\prime \prime}(t)$ \\
\hline$\sigma=\frac{1}{16}$ & 0.3254 & 0.0308 & 0.0123 \\
\hline$\sigma=\sigma_{2}(t)$ & 0.1458 & 0.0264 & 0.0090 \\
\hline
\end{tabular}

Table 1: RMSEs for $z(t)$.

We also consider the recovery results with $\sigma(t) \equiv \sigma=\frac{1}{16}$. Here instead of providing pictures, we use the relative root of mean square error (RMSE) to evaluate the error of component recovery. The RMSE is defined by

$$
\operatorname{RMSE}_{v}:=\frac{1}{K} \sum_{k=1}^{K} \frac{\left\|v_{k}-\widehat{v}_{k}\right\|_{2}}{\left\|v_{k}\right\|_{2}},
$$

where $v$ is a vector and $\widehat{v}$ is an estimation of $v$. Note that as mentioned above, the RMSE is calculated over $[N / 8+1: 7 N / 8]$ to avoid the errors near the end points, which are usually large. We use $\mathrm{ASTFT}_{\mathrm{si}}$ and $\mathrm{ASTFT}_{\mathrm{lc}}$ to denote the sinusoidal signal-based model and the linear chirp-based model respectively. From Table 1, we see the recovery formula based on adaptive STFT with time-varying $\sigma(t)$ results in smaller component recovery errors than that based on the conventional STFT.

\section{References}

[1] R. Behera, S. Meignen, and T. Oberlin, "Theoretical analysis of the 2nd-order synchrosqueezing transform," Appl. Comput. Harmon. Anal., vol. 45, no. 2, pp. 379-404, 2018.

[2] A.J. Berrian and N. Saito, "Adaptive synchrosqueezing based on a quilted short-time Fourier transform," arXiv:1707.03138v5, Sep. 2017.

[3] R.L. Burden and J.D. Faires, Numerical Analysis, (9th Ed.), Brooks/Cole, 2011.

[4] H.Y. Cai, Q.T. Jiang, L. Li and B.W. Suter, "Analysis of adaptive short-time Fourier transform-based synchrosqueezing transform," Analysis and Applications, 2020, in press. https://doi.org/10.1142/S0219530520400047

[5] C.K. Chui, Y.-T. Lin, and H.-T. Wu, "Real-time dynamics acquisition from irregular samples - with application to anesthesia evaluation," Anal. Appl., vol. 14, no. 4, pp. 537-590, 2016.

[6] C.K. Chui, Q.T. Jiang, L. Li, and J. Lu, "A signal separation method based on adaptive continuous wavelet transform and its analysis," preprint, 2020. 
[7] C.K. Chui and N.N. Han, "Wavelet thresholding for recovery of active sub-signals of a composite signal from its discrete samples," preprint, 2020.

[8] C.K. Chui and H.N. Mhaskar, "Signal decomposition and analysis via extraction of frequencies," Appl. Comput. Harmon. Anal., vol. 40, no. 1, pp. 97-136, 2016.

[9] C.K. Chui and H.N. Mhaskar, "Naive instantaneous frequency estimation and signal separation from blind source," preprint, 2020.

[10] C.K. Chui and M.D. van der Walt, "Signal analysis via instantaneous frequency estimation of signal components," Int'l J. Geomath., vol. 6, no. 1, pp. 1-42, 2015.

[11] I. Daubechies, J.F. Lu, and H.-T. Wu, "Synchrosqueezed wavelet transforms: An empirical mode decomposition-like tool," Appl. Comput. Harmon. Anal., vol. 30, no. 2, pp. 243-261, 2011.

[12] I. Daubechies and S. Maes, "A nonlinear squeezing of the continuous wavelet transform based on auditory nerve models," in A. Aldroubi, M. Unser Eds. Wavelets in Medicine and Biology, CRC Press, 1996, pp. 527-546.

[13] D.J. Gibson, "Fourier transform of a linearly-chirped Gaussian pulse," manuscript, 2006, available at: http://archive.physiker.us/files/physics/ChirpedPulseTransform.pdf.

[14] N.E. Huang, Z. Shen, S.R. Long, M.L. Wu, H.H. Shih, Q. Zheng, N.C. Yen, C.C. Tung, and H.H. Liu, "The empirical mode decomposition and Hilbert spectrum for nonlinear and nonstationary time series analysis," Proc. Roy. Soc. London A, vol. 454, no. 1971, pp. 903-995, 1998.

[15] Q.T. Jiang and B.W. Suter, "Instantaneous frequency estimation based on synchrosqueezing wavelet transform," Signal Proc., vol. 138, no. pp. 167-181, 2017.

[16] C. Li and M. Liang, "A generalized synchrosqueezing transform for enhancing signal timefrequency representation," Signal Proc., vol. 92, no. 9, pp. 2264-2274, 2012.

[17] L. Li, H.Y. Cai, H.X. Han, Q.T. Jiang and H.B. Ji, "Adaptive short-time Fourier transform and synchrosqueezing transform for non-stationary signal separation," Signal Proc., vol. 166, 2020, 107231. https://doi.org/10.1016/j.sigpro.2019.07.024

[18] L. Li, H.Y. Cai and Q.T. Jiang, "Adaptive synchrosqueezing transform with a time-varying parameter for non-stationary signal separation," Appl. Comput. Harmon. Anal., vol. 49, 10751106, 2020. 
[19] L. Li, C.K. Chui, and Q.T. Jiang, "Direct signals separation via extraction of local frequencies with adaptive time-varying parameters", preprint, Jan 18, 2020 (submitted to IEEE Trans SP on Jan 21, 2020, ms \# T-SP-26004-2020).

[20] L. Li, N.N. Han, Q.T. Jiang, and C.K. Chui, "A separation method for multicomponent nonstationary signals with crossover instantaneous frequencies", preprint, Feb 8, 2020 (submitted to IEEE Trans IT on Feb 14, 2020, ms \# IT-20-0113).

[21] J. Lu, Q.T. Jiang and L. Li, Analysis of adaptive synchrosqueezing transform with a timevarying parameter, Advance in Computational Mathematics, Vol. 46, Article number: 72 (2020). https://doi.org/10.1007/s10444-020-09814-x

$[22]$ T. Oberlin and S. Meignen, "The 2nd-order wavelet synchrosqueezing transform," in 2017 IEEE International Conference on Acoustics, Speech and Signal Processing (ICASSP), March 2017, New Orleans, LA, USA.

[23] T. Oberlin, S. Meignen, and V. Perrier, "The Fourier-based synchrosqueezing transform," in Proc. 39th Int. Conf. Acoust., Speech, Signal Proc. (ICASSP), 2014, pp. 315-319.

[24] T. Oberlin, S. Meignen, and V. Perrier, "Second-order synchrosqueezing transform or invertible reassignment? Towards ideal time-frequency representations," IEEE Trans. Signal Proc., vol. 63, no. 5, pp. 1335-1344, 2015.

[25] Y.-L. Sheu, L.-Y. Hsu, P.-T. Chou, and H.-T. Wu, "Entropy-based time-varying window width selection for nonlinear-type time-frequency analysis," Int'l J. Data Sci. Anal., vol. 3, pp. 231-245, 2017.

[26] G. Thakur and H.-T. Wu, "Synchrosqueezing based recovery of instantaneous frequency from nonuniform samples," SIAM J. Math. Anal., vol. 43, no. 5, pp. 2078-2095, 2011.

[27] S.B. Wang, X.F. Chen, G.G. Cai, B.Q. Chen, X. Li, and Z.J. He, "Matching demodulation transform and synchrosqueezing in time-frequency analysis," IEEE Trans. Signal Proc., vol. 62 , no. 1, pp. 69-84, 2014.

[28] S.B. Wang, X.F. Chen, I.W. Selesnick, Y.J. Guo, C.W. Tong and X.W. Zhang, "Matching synchrosqueezing transform: A useful tool for characterizing signals with fast varying instantaneous frequency and application to machine fault diagnosis," Mechanical Systems and Signal Proc., vol. 100, pp. 242-288, 2018.

[29] H.Z. Yang, "Synchrosqueezed wave packet transforms and diffeomorphism based spectral analysis for 1D general mode decompositions," Appl. Comput. Harmon. Anal., vol. 39, no.1, pp. 33-66, 2015. 